\title{
Computationally sound implementations of equational theories against passive adversaries ${ }^{\text {th }}$
}

\author{
Mathieu Baudet ${ }^{\mathrm{a}}$, Véronique Cortier ${ }^{\mathrm{b}}$, Steve Kremer $^{\mathrm{c}}$ \\ ${ }^{a}$ DCSSI, France \\ ${ }^{b}$ Loria/CNRS \& INRIA Lorraine projet Cassis, France \\ ${ }^{c} L S V / C N R S \&$ INRIA Saclay projet SECSI \& ENS Cachan, France
}

\begin{abstract}
In this paper we study the link between formal and cryptographic models for security protocols in the presence of passive adversaries. In contrast to other works, we do not consider a fixed set of primitives but aim at results for arbitrary equational theories. We define a framework for comparing a cryptographic implementation and its idealization with respect to various security notions. In particular, we concentrate on the computational soundness of static equivalence, a standard tool in cryptographic pi calculi. We present a soundness criterion, which for many theories is not only sufficient but also necessary. Finally, to illustrate our framework, we establish the soundness of static equivalence for the exclusive OR and a theory of ciphers and lists.
\end{abstract}

\section{Introduction}

Today's ubiquity of computer networks increases the need for theoretic foundations for cryptographic protocols. For more than twenty years now, two communities separately developed two families of models. Both views have been very useful in increasing the understanding and quality of security protocol design. On the one hand formal or logical models have been developed, based on the seminal work of Dolev and Yao [2]. These models view cryptographic operations in a rather abstract and idealized way. On the other hand cryptographic or computational models [3] are closer to implementations: cryptographic operations are modeled as algorithms manipulating bit-strings. Those models cover a large class of attacks, namely all those implementable by a probabilistic polynomial-time Turing machine.

The advantage of formal models is that security proofs are generally simpler and suitable for automatic procedures, even for complex protocols. Unfortunately, the high degree of abstraction and the limited adversary power raise

\footnotetext{
An extended abstract of this work was published in the proceedings of the ICALP'05 conference [1].

Email addresses: mathieu.baudet@sgdn.gouv.fr (Mathieu Baudet), cortier@loria.fr (Véronique Cortier), kremer@lsv.ens-cachan.fr (Steve Kremer)
} 
questions regarding the security offered by such proofs. Potentially, justifying symbolic proofs with respect to standard computational models has tremendous benefits: protocols can be analyzed using automated tools and still benefit from the security guarantees of the computational model.

For the past few years, a significant research effort has been directed at linking these two approaches. In their seminal work [4], Abadi and Rogaway prove the computational soundness of formal (symmetric) encryption in the case a passive attacker. Since then, many results have been obtained. Each of these results considers a fixed set of primitives, for instance symmetric or publickey encryption. In this paper, we aim at presenting general results for arbitrary equational theories, such as encryption, but also less studied ones, such as groups or exclusive OR. The interest of our approach is not only to develop a general and unified framework for the treatment of cryptographic primitives. Conceptually, it also offers a better understanding of the use of equational theories when modeling the algebraic properties of the primitives. Indeed, for several years, formal models have considered equational theories like the theory of exclusive OR, abelian groups or homomorphic encryption (for a survey on algebraic properties see for instance $[5,6]$ ) in order to model some cryptographic aspects. But it is a priori unclear whether "enough" equations have been considered to provide realistic security guarantees. A real attacker might still exploit additional properties of a cryptographic primitive that have not been modeled. Here, we propose a setting and some proof techniques that allow us to formally define and prove that "enough" equations have been considered.

We concentrate on static equivalence, a now standard notion originating from the applied pi calculus [7]. Intuitively, static equivalence asks whether an attacker can distinguish between two tuples of messages - later called frames by exhibiting a relation which holds on one tuple but not on the other. Static equivalence provides an elegant means to express security properties on pieces of data, for instance those observed by a passive attacker during the run of a protocol. In the context of active attackers, static equivalence has also been used to characterize process equivalences [7] and off-line guessing attacks [8, 9]. There now exist exact [10] and approximate [11] algorithms to decide static equivalence for a large family of equational theories.

Our first contribution is a general framework for comparing formal and computational models in the presence of a passive attacker. We define the notions of soundness and faithfulness of a cryptographic implementation with respect to equality, static equivalence and (non-)deducibility. Soundness holds when a formal notion of security has a computational interpretation. For instance, statically equivalent tuples of messages (frames) should be computationally indistinguishable. Conversely, faithfulness holds when every formal attack on a given notion of security can be mapped to an efficient computational attacker. As an illustration, we consider an equational theory modeling Abelian groups with exponents taken over a commutative ring. We show that the soundness of static equivalence implies the hardness of several classical problems in cryptography, notably the decisional Diffie-Hellmann and the RSA problem. Although not completely surprising, this results illustrate well the expressive power of 
static equivalence defined over tailored equational theories.

Our second contribution is a sufficient criterion for soundness with respect to static equivalence: intuitively the usual computational semantics of terms has to be indistinguishable to an idealized one. We also define and study a useful class of frames, called transparent frames, for arbitrary equational theories. Informally, a frame is transparent if every secret in use is deducible from the frame itself. Transparent frames enjoy notable properties such as a simple characterization of static equivalence and - in the case of uniform distributions - the fact that two statically equivalent transparent frames always yield the same concrete distribution, that is, are indistinguishable in the sense of information theory. This study of transparent frames allows us to exhibit a class of equational theories for which our soundness criterion is necessary.

Our third contribution consists in applying our framework to obtain two first soundness results for static equivalence. The first equational theory that we consider deals with the exclusive OR. This simple but important primitive has been largely used in cryptographic constructions such as the One-Time Pad and in protocols (see [6] for examples). Interestingly, our proof of soundness reflects the unconditional security (in the information-theoretic sense) of the One-Time Pad [12]. Second we consider a theory of symmetric encryption and lists. The result is similar in spirit to the one of Abadi and Rogaway [4]. However, we consider deterministic, length-preserving, symmetric encryption schemes - also known as pseudo-random permutations or ciphers, while Abadi and Rogaway consider probabilistic, symmetric encryption. This choice is motivated by famous examples of ciphers such as DES or AES. In both examples, the specificity of our work is to prove the soundness of a standard formal notion, static equivalence, rather than that of a specialized relation.

Related work. The study of the link between the formal and the computational approaches for cryptographic protocols started with the seminal work of Abadi and Rogaway [4], in a passive setting. There have been many extensions to the work of Abadi and Rogaway in the passive case, such as studying completeness [13], considering deterministic encryption [14] (a more detailed comparison is provided below), One-Time pad, length-revealing and same-key revealing encryption [12] or allowing composed keys [15] and key-cycles [16].

The first results in an active setting were achieved by Backes, Pfitzmann, and Waidner $[17,18,19]$. These works prove the soundness of a rich language including digital signatures, public-key and symmetric key encryption in the presence of an active attacker for several kind of security properties. Quite similar results were established in more abstract and classical Dolev-Yao models for asymmetric encryption and signatures $[20,21]$. While more easily amendable to full automation, these results do not offer universal composability guarantees like the previous ones. However, Canetti and Herzog [22] have recently obtained a similar soundness theorem for a restricted class of protocols-mutual authentication and key exchange protocols using only public-key encryptionwhich does offer strong composability properties in the universal composability framework. Laud [23] presents an automated procedure for computationally 
sound proofs of confidentiality in the case of an active attacker and symmetric encryption when the number of sessions is bounded. Datta et al. [24] introduce a symbolic logic that allows cryptographically sound security proof. Recently, Blanchet [25] proposed a computationally sound mechanized prover that relies directly on games transformations, a proof technique commonly used in the cryptographic setting.

Except [25], the previously mentioned results are all dedicated to some fixed set of cryptographic primitives. Here, our goal is not restricted to obtaining some particular soundness result for a given set of primitives and security properties. Rather, we aim at developing a general setting to reason about the adequacy of abstract functional symbols equipped with an equational theory and their corresponding cryptographic implementations. To the best of our knowledge, this approach is new and distinct from existing work. We now discuss some related work concerning the two theories (exclusive OR as well as ciphers and lists) that we have considered to illustrate our framework.

Regarding the soundness of exclusive OR, Backes and Pfitzmann [26] have independently shown an impossibility result in the framework of reactive simulatability, in the presence of an active adversary. They also present a soundness result in the presence of a passive adversary. While we consider the application of exclusive OR only to pure random values, Backes and Pfitzmann deal with arbitrary payloads. It is however not clear how the framework of reactive simulatability in the presence of a passive adversary compares to our framework based on static equivalence.

Concerning the theory of ciphers and list, Laud [14] presents soundness results in the style of Abadi and Rogaway for ciphers. While these results are close to ours, Laud's notion of formal equivalence is apparently more pessimistic than ours regarding the secrecy of encryption keys. For instance, as opposed to [14], we consider that the encryption of a fresh random value by a known key is indistinguishable from a random value - that is, formally, the pair (enc $(n, k), k)$ is indistinguishable from $\left(n^{\prime}, k\right)$. The reason is that, in the absence of tags, each encryption key of a cipher yields a permutation on the space of values. Therefore, if $n$ follows the uniform distribution, such as in our implementation (Section 5.2), so does the term enc $(n, k)$. Provided a suitable set of equations, static equivalence naturally accounts for this property, whereas there seems to be no natural and immediate way to express the same equivalences using patterns in the style of Abadi and Rogaway. In some sense, the work of Abadi and Warinschi [27] can be seen as an attempt to do so on a fragment of equivalences modeling guessing attacks. Recently, the techniques developed in the present paper have been applied successfully by Abadi, Baudet, and Warinschi [28] to generalize the ideas of [27] and justify a modeling of guessing attacks purely based on static equivalence.

In [14], Laud provides a computationally sound proof system handling both ciphers and exclusive OR in the presence of a passive attacker. This proof system is used to prove the security of several encryption modes including CBC. This approach differs from the one developed here as it aims at direct cryptographic proofs of security (much as in $[23,25]$ ). In comparison, our approach (as in [4, 
$12,15,16,17,18,19,13,20,21])$ aims to exhibit a class of protocols for which the absence of formal attacks entails the existence of a computational proof of security.

Further related work. Since the publication of a preliminary version [1] of this article, several papers have addressed the computational soundness of static equivalence. As already mentioned, Abadi, Baudet, and Warinschi [28] study resistance against offline guessing attacks modelled in terms of static equivalence and use the framework developed in this paper to show the soundness of an equational theory including ciphers, symmetric and asymmetric encryption. In [29], Bana, Mohassel and Stegers argue that the notion of static equivalence is too coarse and not sound for many interesting equational theories. They introduce a general notion of formal indistinguishability relation. This highlights that soundness of static equivalence only holds for a restricted set of well-formed frames (in the same vein Abadi and Rogaway used restrictions to forbid key cycles). They illustrate the unsoundness of static equivalence for modular exponentiation. More recently, Kremer and Mazaré [30] use our framework to define soundness of static equivalence in the presence of an adaptive, rather than purely passive, adversary. They show soundness results of static equivalence for an equational theory modelling modular exponentiation (for a class of well-formed frames, hence not contradicting [29]), as well as symmetric encryption with composed keys which can be computed using modular exponentiation or exclusive or.

The active version of static equivalence is the observational equivalence relation introduced by Milner and Hoare in the early 80s. Intuitively, two processes are equivalent if an observer cannot tell the difference between the two processes. The observer can in particular intercept and send messages to the processes. Comon-Lundh and Cortier [31] have recently shown that observational equivalence between processes in a fragment of the applied pi-calculus [32] implies cryptographic indistinguishability against active attackers, in the context of symmetric encryption. They use an extended version of soundness of static equivalence (called tree soundness) as a key step in their proof.

Outline of the paper.. In the next section, we introduce our abstract and concrete models together with the notions of indistinguishability. We then define the notions of soundness and faithfulness and illustrate some consequences of soundness with respect to static equivalence on groups. In Section 4, we define the ideal semantics of abstract terms, present our soundness criterion, and prove it necessary for a large family of equational theories. As an illustration (Section 5), we prove the soundness for the theories modeling exclusive OR, as well as ciphers and lists. We then conclude in Section 6. An appendix contains detailed proofs of formal lemmas related to static equivalence.

\section{Modeling cryptographic primitives with abstract algebras}

In this section we introduce some notations and set our abstract and concrete models. 


\subsection{Abstract algebras}

Our abstract models - called abstract algebras - consist of term algebras defined over a many-sorted first-order signature and equipped with equational theories.

Specifically, a signature $(\mathcal{S}, \mathcal{F})$ is made of a set of sorts $\mathcal{S}$, with elements denoted by $s, s_{1} \ldots$, and a set $\mathcal{F}$ of symbols, written $f, f_{1} \ldots$, together with arities of the form $\operatorname{ar}(f)=s_{1} \times \ldots \times s_{k} \rightarrow s(k \geq 0)$. Symbols that take $k=0$ arguments are called constants; their arity is simply written $s$. We fix a set $\mathcal{N}$ of names, written $a, b \ldots$, and a set $\mathcal{X}$ of variables $x, y \ldots$ We assume that names and variables are given with sorts, and that an infinite number of names and variables are available for each sort. The set of terms of sort $s$ is defined inductively by

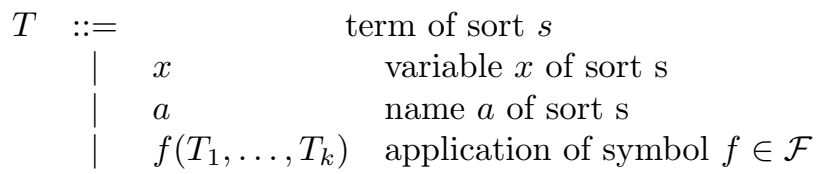

where for the last case, we further require that $T_{i}$ is a term of some sort $s_{i}$ and $\operatorname{ar}(f)=s_{1} \times \ldots \times s_{k} \rightarrow s$. We write $\operatorname{var}(T)$ and names $(T)$ for the set of variables and names occurring in $T$, respectively. A term $T$ is ground or closed iff $\operatorname{var}(T)=\emptyset$. We may write $\operatorname{var}\left(T_{1}, \ldots, T_{k}\right)$ instead of $\operatorname{var}\left(\left\{T_{1}, \ldots, T_{k}\right\}\right)$ and similarly for names.

A context $C$ is a term with holes, or (more formally) a term with distinguished variables. When $C$ is a context with $n$ distinguished variables $x_{1}, \ldots$, $x_{n}$, we may write $C\left[x_{1}, \ldots, x_{n}\right]$ instead of $C$ in order to show the variables, and when $T_{1}, \ldots, T_{n}$ are terms we may also write $C\left[T_{1}, \ldots, T_{n}\right]$ for the result of replacing each variable $x_{i}$ with the corresponding term $T_{i}$.

Substitutions are written $\sigma=\left\{x_{1} \mapsto T_{1}, \ldots, x_{n} \mapsto T_{n}\right\}$ with domain $\operatorname{dom}(\sigma)=$ $\left\{x_{1}, \ldots, x_{n}\right\}$. We only consider well-sorted substitutions, that is, substitutions $\sigma=\left\{x_{1} \mapsto T_{1}, \ldots, x_{n} \mapsto T_{n}\right\}$ for which $x_{i}$ and $T_{i}$ have the same sort. Such a $\sigma$ is closed iff all of the $T_{i}$ are closed. We let $\operatorname{var}(\sigma)=\bigcup_{i} \operatorname{var}\left(T_{i}\right)$, $\operatorname{names}(\sigma)=\bigcup_{i}$ names $\left(T_{i}\right)$, and extend the notations $\operatorname{var}($.$) and names(.) to tu-$ ples and sets of terms and substitutions in the obvious way. The application of a substitution $\sigma$ to a term $T$ is written $\sigma(T)=T \sigma$. If $p$ is a position of $T$, the expression $\left.T\right|_{p}$ denotes the subterm of $T$ at the position $p$. The expression $T\left[T^{\prime}\right]_{p}$ denotes the term obtained after replacing the subterm in position $p$ of $T$ with $T^{\prime}$.

Symbols in $\mathcal{F}$ are intended to model cryptographic primitives, whereas names in $\mathcal{N}$ are used to model secrets, that is, concretely random numbers. The intended behavior of the primitives is described by an equational theory $E$, that is, an equivalence relation on terms (also written $=_{E}$ ) compatible with applications of symbols and well-sorted substitutions:

- for every $k$-ary symbol $f$ and terms $t_{1}, \ldots, t_{k}, t_{1}^{\prime}, \ldots, t_{k}^{\prime}$ of the appropriate sorts, $\forall i, t_{i}={ }_{E} t_{i}^{\prime}$ implies that $f\left(t_{1}, \ldots, f_{k}\right)={ }_{E} f\left(t_{1}^{\prime}, \ldots, f_{k}^{\prime}\right)$; 
- for every well-sorted substitution $\sigma$ and terms $t, t^{\prime}$, if $t={ }_{E} t^{\prime}$ then $t \sigma={ }_{E}$ $t^{\prime} \sigma$.

In the sequel we further require that $E$ is stable under (well-sorted) substitution of names. All the equational theories that we consider in this paper satisfy these properties. For instance, symmetric and deterministic encryption is modeled by the theory $E_{\text {enc }}$ generated by the classical equation $E_{\mathrm{enc}}=\{\operatorname{dec}(\operatorname{enc}(x, y), y)=x\}$.

A symbol $f$ is free with respect to an equational theory $E$ iff there exists a set of equations $F$ generating $E$ such that $f$ does not occur in $F$. A sort $s$ is degenerated in $E$ iff all terms of sort $s$ are equal modulo $E$.

It is often useful to orient equations and work with rewriting rules instead of the equational theory. Formally, a rewriting rule is an expression $l \rightarrow r$ where $l$ and $r$ are two terms of the same sort. Given a set of rewriting rules $\mathcal{R}$ (called rewriting system), we write $T \rightarrow_{\mathcal{R}} T^{\prime}$ if there exists a rule $l \rightarrow r \in \mathcal{R}$, a position $p$ and a (well-sorted) substitution $\sigma$ such that $\left.T\right|_{p}=l \sigma$ and $T^{\prime}=T[r \sigma]_{p}$. We write $\rightarrow_{\mathcal{R}}^{*}$ for the reflexive and transitive closure of $\rightarrow_{\mathcal{R}}$, and $=_{\mathcal{R}}$ for its reflexive, symmetric and transitive closure.

Given an equational theory $E$ and a rewriting system $\mathcal{R}$, we write $\rightarrow_{\mathcal{R} / E}$ for the relation $={ }_{E} \rightarrow_{\mathcal{R}}={ }_{E}$. We define $\rightarrow_{\mathcal{R} / E}^{*}$ and $=_{\mathcal{R} / E}$ similarly as above. $\mathcal{R}$ is $E$-terminating iff $\rightarrow_{\mathcal{R} / E}$ admits no infinite sequence of reductions $T_{0} \rightarrow_{\mathcal{R} / E}$ $T_{1} \rightarrow_{\mathcal{R} / E} \ldots T_{n} \rightarrow_{\mathcal{R} / E} \ldots$. It is E-confluent iff for every $T \rightarrow_{\mathcal{R} / E}^{*} T_{1}$ and $T \rightarrow_{\mathcal{R} / E}^{*} T_{2}$, there exist $T_{1}^{\prime}$ and $T_{2}^{\prime}$ such that $T_{1} \rightarrow_{\mathcal{R} / E}^{*} T_{1}^{\prime}, T_{2} \rightarrow_{\mathcal{R} / E}^{*} T_{2}^{\prime}$, and $T_{1}^{\prime}={ }_{E} T_{2}^{\prime}$. Finally, $\mathcal{R}$ is $E$-convergent iff it is both $E$-terminating and $E$-confluent. When $E$ is the syntactic equality, this yields the usual notions of termination, confluence and convergence.

\subsection{Frames, deducibility and static equivalence}

We use frames $[7,10]$ to represent sequences of messages observed by an attacker, for instance during the execution of a protocol. Formally, a (closed) frame is an expression $\varphi=\nu \tilde{a} .\left\{x_{1}=T_{1}, \ldots, x_{n}=T_{n}\right\}$ where $\tilde{a}$ is a set of bound (or restricted) names, and for each $i, T_{i}$ is a closed term of the same sort as $x_{i}$.

For simplicity, we only consider (closed) frames $\varphi=\nu \tilde{a} .\left\{x_{1}=T_{1}, \ldots, x_{n}=\right.$ $\left.T_{n}\right\}$ which restrict every name in use, that is, for which $\tilde{a}=\operatorname{names}\left(T_{1}, \ldots, T_{n}\right)$. A name $a$ may still be disclosed explicitly by adding a mapping $x_{a}=a$ to the frame. Thus we tend to assimilate such frames $\varphi$ to their underlying substitutions $\sigma=\left\{x_{1} \mapsto T_{1}, \ldots, x_{n} \mapsto T_{n}\right\}$.

Definition 1 (Deducibility). A (closed) term $T$ is deducible from a frame $\varphi$ in an equational theory $E$, written $\varphi \vdash_{E} T$, iff there exists a term $M$ such that $\operatorname{var}(M) \subseteq \operatorname{dom}(\varphi)$, names $(M) \cap \operatorname{names}(\varphi)=\emptyset$, and $M \varphi={ }_{E} T$.

In what follows, again for simplicity, we only consider deducibility problems $\varphi \vdash_{E} T$ such that names $(T) \subseteq \operatorname{names}(\varphi)$.

Consider for instance the theory $E_{\text {enc }}$ and the frame $\varphi_{1}=\nu k_{1}, k_{2}, k_{3}, k_{4} \cdot\left\{x_{1}=\right.$ enc $\left.\left(k_{1}, k_{2}\right), x_{2}=\operatorname{enc}\left(k_{4}, k_{3}\right), x_{3}=k_{3}\right\}$ : the name $k_{4}$ is deducible from $\varphi_{1}$ since $\operatorname{dec}\left(x_{2}, x_{3}\right) \varphi_{1}={ }_{E_{\text {enc }}} k_{4}$ but neither $k_{1}$ nor $k_{2}$ are deducible. 
Deducibility is not always sufficient to account for the knowledge of an attacker. For instance, it lacks partial information on secrets. Indeed, if we consider a naive vote protocol where agents simply send their vote (0 or 1$)$ encrypted under some key, the security problem is not whether an attacker can learn the values of 0 or 1 , but rather whether an attacker can tell the difference between a message that contains the vote 0 and a message that contains the vote 1. That is why another classical notion in formal methods is static equivalence.

Definition 2 (Static equivalence). Two frames $\varphi_{1}$ and $\varphi_{2}$ are statically equivalent in a theory $E$, written $\varphi_{1} \approx_{E} \varphi_{2}$, iff $\operatorname{dom}\left(\varphi_{1}\right)=\operatorname{dom}\left(\varphi_{2}\right)$, and for all terms $M$ and $N$ such that $\operatorname{var}(M, N) \subseteq \operatorname{dom}\left(\varphi_{1}\right)$ and names $(M, N) \cap \operatorname{names}\left(\varphi_{1}, \varphi_{2}\right)=$ $\emptyset, M \varphi_{1}={ }_{E} N \varphi_{1}$ if and only if $M \varphi_{2}={ }_{E} N \varphi_{2}$.

For instance, the two frames $\nu k .\{x=\operatorname{enc}(0, k)\}$ and $\nu k .\{x=\operatorname{enc}(1, k)\}$ are statically equivalent with respect to $E_{\text {enc }}$. However the two frames

$$
\nu k .\{x=\operatorname{enc}(0, k), y=k\} \text { and } \nu k \cdot\{x=\operatorname{enc}(1, k), y=k\}
$$

are not (consider the test $\operatorname{dec}(x, y) \stackrel{?}{=} 0$ ), although the set of terms that can be deduced from both frames is the same ( 0 and 1 are two constants known by the attacker).

\subsection{Concrete semantics}

We now give terms and frames a concrete semantics, parameterized by an implementation of the primitives. Provided a set of sorts $\mathcal{S}$ and a set of symbols $\mathcal{F}$ as above, a $(\mathcal{S}, \mathcal{F})$-computational algebra $A$ consists of

- a non-empty set of bit-strings $\llbracket s \rrbracket_{A} \subseteq\{0,1\}^{*}$ for each sort $s \in \mathcal{S}$;

- an effective procedure implementing a function $\llbracket f \rrbracket_{A}: \llbracket s_{1} \rrbracket_{A} \times \ldots \times \llbracket s_{k} \rrbracket_{A} \rightarrow$ $\llbracket s \rrbracket_{A}$ for each symbol $f \in \mathcal{F}$ with $\operatorname{ar}(f)=s_{1} \times \ldots \times s_{k} \rightarrow s$;

- an effective procedure for deciding a congruence $=_{A, s}$ for each sort $s$, in order to check the equality of elements in $\llbracket s \rrbracket_{A}$ (the same element may be represented by different bit-strings); by congruence, we mean a reflexive, symmetric, transitive relation such that $e_{1}={ }_{A, s_{1}} e_{1}^{\prime}, \ldots, e_{k}={ }_{A, s_{k}} e_{k}^{\prime} \Rightarrow$ $\llbracket f \rrbracket_{A}\left(e_{1}, \ldots, e_{k}\right)={ }_{A, s} \llbracket f \rrbracket_{A}\left(e_{1}^{\prime}, \ldots, e_{k}^{\prime}\right)$ (in the remaining we often omit $s$ and write $=_{A}$ for $=_{A, s}$;

- an effective procedure to draw random elements from $\llbracket s \rrbracket_{A}$; we denote such a drawing by $x \stackrel{R}{\longleftarrow} \llbracket s \rrbracket_{A}$; the drawing may not follow a uniform distribution, but no $=_{A, s}$-equivalence class should have probability 0 .

Assume a fixed $(\mathcal{S}, \mathcal{F})$-computational algebra $A$. We associate to each (closed) frame $\varphi=\left\{x_{1}=T_{1}, \ldots, x_{n}=T_{n}\right\}$ a distribution $\psi=\llbracket \varphi \rrbracket_{A}$, of which the drawings $\widehat{\psi} \stackrel{R}{\longleftarrow} \psi$ are computed as follows:

1. for each name $a$ of sort $s$ appearing in $T_{1}, \ldots, T_{n}$, draw a value $\widehat{a} \stackrel{R}{\longleftarrow} \llbracket s \rrbracket_{A}$; 
2. for each $x_{i}(1 \leq i \leq n)$ of sort $s_{i}$, compute $\widehat{T}_{i} \in \llbracket s_{i} \rrbracket_{A}$ recursively on the structure of terms: $f\left(\widehat{T_{1}^{\prime}, \ldots,}, T_{m}^{\prime}\right)=\llbracket f \rrbracket_{A}\left(\widehat{T_{1}^{\prime}}, \ldots, \widehat{T_{m}^{\prime}}\right)$; using the values $\widehat{a}$ defined at step 1 for names.

3. return the value $\widehat{\psi}=\left\{x_{1}=\widehat{T_{1}}, \ldots, x_{n}=\widehat{T_{n}}\right\}$.

Such values $\phi=\left\{x_{1}=e_{1}, \ldots, x_{n}=e_{n}\right\}$ with $e_{i} \in \llbracket s_{i} \rrbracket_{A}$ are called concrete frames. We extend the notation $\llbracket \cdot \rrbracket_{A}$ to tuples of closed terms in the natural way: $e_{1}, \ldots, e_{n} \stackrel{R}{\longleftarrow} \llbracket T_{1}, \ldots, T_{n} \rrbracket_{A}$ denotes the drawing

$$
\left\{x_{1}=e_{1}, \ldots, x_{n}=e_{n}\right\} \stackrel{R}{\longleftarrow} \llbracket\left\{x_{1}=T_{1}, \ldots, x_{n}=T_{n}\right\} \rrbracket_{A}
$$

for appropriate variables $x_{1}, \ldots, x_{n}$. We also generalize the notation to (tuples of) terms with variables, by specifying a concrete value for each of them: $\llbracket . \rrbracket_{A,\left\{x_{1}=e_{1}, \ldots, x_{n}=e_{n}\right\}}$. Notice that when a term or a frame contains no names, the translation is deterministic; in this case, we use the same notation to denote the distribution and its unique value.

In the rest of the paper we focus on asymptotic notions of cryptographic security and consider families of computational algebra $\left(A_{\eta}\right)$ indexed by a complexity parameter $\eta \geq 0$. (This parameter $\eta$ might be thought as the size of keys and other secret values.) The concrete semantics of a frame $\varphi$ is a family of distributions over concrete frames $\left(\llbracket \varphi \rrbracket_{A_{\eta}}\right)$. We only consider families of computational algebras $\left(A_{\eta}\right)$ such that the algebraic operations (i.e. the functions associated to symbols, the congruence relation $=_{A, s}$, and the drawing functions) are computable by uniform, probabilistic polynomial-time algorithms in the complexity parameter $\eta$. This ensures that the concrete semantics of every (fixed) term or frame is efficiently computable (in the same sense).

Families of distributions (ensembles) over concrete frames benefit from the usual notion of cryptographic indistinguishability. Intuitively, two families of distributions $\left(\psi_{\eta}\right)$ and $\left(\psi_{\eta}^{\prime}\right)$ are indistinguishable, written $\left(\psi_{\eta}\right) \approx\left(\psi_{\eta}^{\prime}\right)$, iff no probabilistic polynomial-time adversary $\mathcal{A}$ can guess whether he is given a sample from $\psi_{\eta}$ or $\psi_{\eta}^{\prime}$ with a probability significantly greater than $\frac{1}{2}$. Formally, we ask the advantage of $\mathcal{A}$,

$$
\operatorname{Adv}^{\mathrm{IND}}\left(\mathcal{A}, \eta, \psi_{\eta}, \psi_{\eta}^{\prime}\right)=\mathbb{P}\left[\widehat{\psi} \stackrel{R}{\longleftarrow} \psi_{\eta}: \mathcal{A}(\eta, \widehat{\psi})=1\right]-\mathbb{P}\left[\widehat{\psi} \stackrel{R}{\longleftarrow} \psi_{\eta}^{\prime}: \mathcal{A}(\eta, \widehat{\psi})=1\right]
$$

to be a negligible function of $\eta$. We recall that a function $f$ is said negligible if for any integer $n>0$, there exists $\eta_{0}$ such that $f(\eta) \leq \eta^{-n}$ for any $\eta \geq \eta_{0}$. (Note that we regard negative functions as negligible here.)

A function $f(\eta)$ is overwhelming iff $1-f(\eta)$ is negligible. A family of distributions $\left(\psi_{\eta}\right)$ is collision-free (with respect to the family of congruences $=_{A_{\eta}}$ ) iff the probability of collision between two random elements from $\psi_{\eta}$, that is, $\mathbb{P}\left[e_{1}, e_{2} \stackrel{R}{\longleftarrow} \psi_{\eta}: e_{1}=A_{A_{\eta}} e_{2}\right]$, is a negligible function of $\eta$. Note that, by classical properties of probability, this is equivalent to requiring that the probability of sampling any given $e_{0}$ from $\psi_{\eta}\left(\right.$ modulo $={ }_{A_{\eta}}$ ) is negligible, that is, the function $\sup _{e_{0}} \mathbb{P}\left[e \stackrel{R}{\longleftarrow} \psi_{\eta}: e=_{A_{\eta}} e_{0}\right]$ is bounded by a negligible function of $\eta$. 
By convention, the adversaries considered in this paper are given access implicitly to the complexity parameter $\eta$ and to as many fresh random coins as needed.

\section{Comparing abstract and computational algebras}

In the previous section we have defined abstract and computational algebras. We now relate formal notions such as equality, (non-)deducibility and static equivalence to their computational counterparts, that is, equality, one-wayness and indistinguishability.

\subsection{Soundness and faithfulness}

We introduce the notions of sound and faithful computational algebras with respect to the formal relations studied here: equality, static equivalence and deducibility.

Let $E$ be an equational theory. A family of computational algebras $\left(A_{\eta}\right)$ is

- ${ }_{E}$-sound iff for every closed terms $T_{1}, T_{2}$ of the same sort, $T_{1}={ }_{E} T_{2}$ implies that $\mathbb{P}\left[e_{1}, e_{2} \stackrel{R}{\longleftarrow} \llbracket T_{1}, T_{2} \rrbracket_{A_{\eta}}: e_{1}={ }_{A_{\eta}} e_{2}\right]$ is overwhelming;

- ${ }_{E}$-faithful iff for every closed terms $T_{1}, T_{2}$ of the same sort, $T_{1} \neq_{E} T_{2}$ implies that $\mathbb{P}\left[e_{1}, e_{2} \stackrel{R}{\longleftarrow} \llbracket T_{1}, T_{2} \rrbracket_{A_{\eta}}: e_{1}={ }_{A_{\eta}} e_{2}\right]$ is negligible;

- $\approx_{E}$-sound iff for every frames $\varphi_{1}, \varphi_{2}$ with the same domain, $\varphi_{1} \approx_{E} \varphi_{2}$ implies that $\left(\llbracket \varphi_{1} \rrbracket_{A_{\eta}}\right) \approx\left(\llbracket \varphi_{2} \rrbracket_{A_{\eta}}\right)$;

- $\approx_{E}$-faithful iff for every frames $\varphi_{1}, \varphi_{2}$ of the same domain, $\varphi_{1} \not_{E} \varphi_{2}$ implies that there exists a polynomial-time adversary $\mathcal{A}$ for distinguishing concrete frames, such that $\operatorname{Adv}^{\operatorname{IND}}\left(\mathcal{A}, \eta, \llbracket \varphi_{1} \rrbracket_{A_{\eta}}, \llbracket \varphi_{2} \rrbracket_{A_{\eta}}\right)$ is overwhelming;

- $\forall_{E}$-sound iff for every frame $\varphi$ and closed term $T$ such that names $(T) \subseteq$ names $(\varphi), \varphi \forall_{E} T$ implies that for each polynomial-time adversary $\mathcal{A}$, $\mathbb{P}\left[\phi, e \stackrel{R}{\longleftarrow} \llbracket \varphi, T \rrbracket_{A_{\eta}}: \mathcal{A}(\phi)={ }_{A_{\eta}} e\right]$ is negligible;

- $\nvdash_{E}$-faithful iff for every frame $\varphi$ and closed term $T$ such that names $(T) \subseteq$ names $(\varphi), \varphi \vdash_{E} T$ implies that there exists a polynomial-time adversary $\mathcal{A}$ such that $\mathbb{P}\left[\phi, e \stackrel{R}{\longleftarrow} \llbracket \varphi, T \rrbracket_{A_{\eta}}: \mathcal{A}(\phi)={ }_{A_{\eta}} e\right]$ is overwhelming.

Sometimes, it is possible to prove stronger notions of soundness that hold without restriction on the computational power of adversaries. In particular, $\left(A_{\eta}\right)$ is

- unconditionally $=_{E}$-sound iff for every closed terms $T_{1}, T_{2}$ of the same sort, $T_{1}={ }_{E} T_{2}$ implies that $\mathbb{P}\left[e_{1}, e_{2} \stackrel{R}{\longleftarrow} \llbracket T_{1}, T_{2} \rrbracket_{A_{\eta}}: e_{1}={ }_{A_{\eta}} e_{2}\right]=1$;

- unconditionally $\approx_{E}$-sound iff for every frames $\varphi_{1}, \varphi_{2}$ with the same domain, $\varphi_{1} \approx_{E} \varphi_{2}$ implies $\left(\llbracket \varphi_{1} \rrbracket_{A_{\eta}}\right)=\left(\llbracket \varphi_{2} \rrbracket_{A_{\eta}}\right)$; 
- unconditionally $\nvdash_{E}$-sound iff for every frame $\varphi$ and closed term $T$ such that names $(T) \subseteq \operatorname{names}(\varphi)$ and $\varphi \nvdash_{E} T$, the drawings for $\varphi$ and $T$ are independent: for all $\phi_{0}, e_{0}, \mathbb{P}\left[\phi_{0}, e_{0} \stackrel{R}{\longleftarrow} \llbracket \varphi, T \rrbracket_{A_{\eta}}\right]=\mathbb{P}\left[\phi_{0} \stackrel{R}{\longleftarrow} \llbracket \varphi \rrbracket_{A_{\eta}}\right] \times$ $\mathbb{P}\left[e_{0} \stackrel{R}{\longleftarrow} \llbracket T \rrbracket_{A_{\eta}}\right]$, and the drawing $\left(\stackrel{R}{\longleftarrow} \llbracket T \rrbracket_{A_{\eta}}\right)$ is collision-free.

The fact that the first two unconditional notions are stronger than their computational counterparts is clear from the definitions. As for the unconditional $\forall E$-soundness, observe that if the drawings for $\varphi$ and $T$ are independent, and the drawing $\left(\stackrel{R}{\longleftarrow} \llbracket T \rrbracket_{A_{\eta}}\right.$ ) is collision-free, then any adversary $\mathcal{A}$ has negligible probability of retrieving the value of $T$ :

$$
\begin{aligned}
& \mathbb{P}\left[\phi, e \stackrel{R}{\longleftarrow} \llbracket \varphi, T \rrbracket_{A_{\eta}}: \mathcal{A}(\phi)={ }_{A_{\eta}} e\right] \\
&=\mathbb{P}\left[\phi \stackrel{R}{\longleftarrow} \llbracket \varphi \rrbracket_{A_{\eta}}, e \stackrel{R}{\longleftarrow} \llbracket T \rrbracket_{A_{\eta}}: \mathcal{A}(\phi)={ }_{A_{\eta}} e\right] \\
& \quad \leq \sup _{e_{0}} \mathbb{P}\left[e \stackrel{R}{\longleftarrow} \llbracket T \rrbracket_{A_{\eta}}: e={ }_{A_{\eta}} e_{0}\right]
\end{aligned}
$$

Generally, (unconditional) ${ }_{E}{ }_{E}$-soundness is given by construction. Indeed true formal equations correspond to the expected behavior of primitives and should hold in the concrete world with overwhelming probability. The other criteria are however more difficult to fulfill. Therefore it is often interesting to restrict frames to well-formed ones in order to achieve soundness or faithfulness: for instance Abadi and Rogaway [4] do forbid encryption cycles (see Section 5.2).

It is worth noting that the notions of soundness and faithfulness introduced above are not independent.

Proposition 1. Let $\left(A_{\eta}\right)$ be $a={ }_{E}$-sound family of computational algebras. Then

1. $\left(A_{\eta}\right)$ is $\forall_{E}$-faithful;

2. if $\left(A_{\eta}\right)$ is also $=_{E}$-faithful, $\left(A_{\eta}\right)$ is $\approx_{E}$-faithful.

Proof.

1. Suppose names $(T) \subseteq$ names $(\varphi)$ and $\varphi \vdash_{E} T$, that is, there exists $M$ such that $\operatorname{var}(M) \subseteq \operatorname{dom}(\varphi)$, names $(M) \cap \operatorname{names}(\varphi)=\emptyset$, and $M \varphi={ }_{E} T$. We define an adversary $\mathcal{A}$ which can deduce $\llbracket T \rrbracket$ from $\llbracket \varphi \rrbracket$ as follows: given the concrete frame $\phi=\left\{x_{i}=e_{i}\right\}, \mathcal{A}$ returns a sample $e \stackrel{R}{\longleftarrow} \llbracket M \rrbracket_{A_{\eta}, \phi}$.

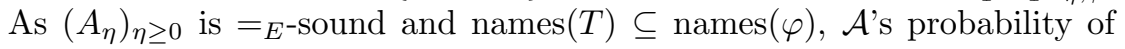
success is greater than 1 minus a negligible function.

2. Suppose $\varphi_{1} \not \varpi_{E} \varphi_{2}$ : there exist two terms $M$ and $N$ such that $\operatorname{var}(M, N) \subseteq$ $\operatorname{dom}\left(\varphi_{1}\right)$, names $(M, N) \cap \operatorname{names}\left(\varphi_{1}, \varphi_{2}\right)=\emptyset$, and for instance $M \varphi_{1}={ }_{E}$ $N \varphi_{1}$ whereas $M \varphi_{2} \neq_{E} N \varphi_{2}$. Let $\mathcal{A}$ be the adversary that tests, given $\eta$ and $\phi$, whether $\llbracket M \rrbracket_{A_{\eta}, \phi}=A_{\eta} \llbracket N \rrbracket_{A_{\eta}, \phi}$, and returns the result of the test. $\mathcal{A}$ runs in polynomial-time and by $={ }_{E}$-soundness and $={ }_{E}$-faithfulness, its advantage is 1 minus a negligible function. 
For many theories, we have that $\approx_{E}$-soundness implies all the other notions of soundness and faithfulness. This emphasizes the importance of $\approx_{E \text {-soundness }}$ and provides an additional motivation for its study. As an illustration, let us consider an arbitrary theory which includes keyed hash functions.

Proposition 2. Let $\left(A_{\eta}\right)$ be a family of $\approx_{E}$-sound computational algebras. Assume that free binary symbols $\mathrm{h}_{s}: s \times$ Key $\rightarrow$ Hash are available for every sort $s$, where the sort Key is not degenerated in $E$, and the drawing of random elements for the sort Hash, $\left(\stackrel{R}{\longleftarrow} \llbracket H a s h \rrbracket_{A_{\eta}}\right)$, is collision-free. Then

1. $\left(A_{\eta}\right)$ is $={ }_{E}$-faithful;

2. $\left(A_{\eta}\right)$ is $\nvdash_{E}$-sound;

3. Assume the implementations for the symbols $\mathrm{h}_{s}$ are collision-resistant, that is, assume that for all $T_{1}, T_{2}$ of sort s, given a fresh name $k$ of sort Key, the quantity

$$
\mathbb{P}\left[e_{1}, e_{2}, e_{1}^{\prime}, e_{2}^{\prime} \stackrel{R}{\longleftarrow} \llbracket T_{1}, T_{2}, \mathrm{~h}_{s}\left(T_{1}, k\right), \mathrm{h}_{s}\left(T_{2}, k\right) \rrbracket_{A_{\eta}}: e_{1} \neq \neq_{A_{\eta}} e_{2}, e_{1}^{\prime}=A_{\eta} e_{2}^{\prime}\right]
$$

is negligible. Then $\left(A_{\eta}\right)$ is $=_{E}$-sound, $\forall_{E}$-faithful and $\approx_{E}$-faithful.

\section{ProOF.}

1. Let $T_{1}, T_{2}$ be two terms of sort $s$ such that $T_{1} \neq_{E} T_{2}$. Consider the frame $\varphi=\left\{x_{1}=\mathrm{h}_{s}\left(T_{1}, k\right), x_{2}=\mathrm{h}_{s}\left(T_{2}, k\right)\right\}$ where $k$ is a fresh name of sort Key. As $T_{1} \neq_{E} T_{2}$ and $\mathrm{h}_{s}$ is free, we have $\varphi \approx_{E}\left\{x_{1}=n, x_{2}=n^{\prime}\right\}$ where $n, n^{\prime}$ are two distinct fresh names of sort Hash (Proposition 17 of Appendix A). By assumption, this entails $\llbracket \varphi \rrbracket \approx \llbracket\left\{x_{1}=n, x_{2}=n^{\prime}\right\} \rrbracket$. In particular, since $\left(\stackrel{R}{\longleftarrow} \llbracket H a s h \rrbracket_{A_{\eta}}\right)$ is collision-free, the quantity

$$
\begin{aligned}
\mathbb{P}\left[e_{1}, e_{2} \stackrel{R}{\longleftarrow} \llbracket T_{1},\right. & \left.T_{2} \rrbracket_{A_{\eta}}: e_{1}={ }_{A_{\eta}} e_{2}\right] \\
& \leq \mathbb{P}\left[e_{1}^{\prime}, e_{2}^{\prime} \stackrel{R}{\longleftarrow} \llbracket \mathrm{h}_{s}\left(T_{1}, k\right), \mathrm{h}_{s}\left(T_{2}, k\right) \rrbracket_{A_{\eta}}: e_{1}^{\prime}={ }_{A_{\eta}} e_{2}^{\prime}\right]
\end{aligned}
$$

is negligible.

2. Let $\varphi$ be a frame and $T$ a closed term of sort $s$ such that names $(T) \subseteq$ names $(\varphi)$ and $\varphi \nvdash_{E} T$. We let $\varphi_{0}=\varphi \cup\left\{x=\mathrm{h}_{s}(T, k), y=k\right\}$ and $\varphi_{1}=\varphi \cup\{x=n, y=k\}$ where $x, y$ are fresh variables, $k$ is a fresh name of sort Key, $n$ is a fresh name of sort Hash. As $\varphi \nvdash_{E} T$, we have $\varphi_{0} \approx_{E} \varphi_{1}$ (Proposition 18 of Appendix A). Thus by assumption, $\llbracket \varphi_{0} \rrbracket \approx \llbracket \varphi_{1} \rrbracket$.

By contradiction, suppose that there exists a polynomial-time adversary $\mathcal{A}$ able to deduce $\llbracket T \rrbracket$ from $\llbracket \varphi \rrbracket$ concretely with non-negligible probability of success. We build an adversary $\mathcal{B}$ that distinguishes between $\llbracket \varphi_{0} \rrbracket$ and $\llbracket \varphi_{1} \rrbracket$ as follows: let $\phi$ be the sample from $\llbracket \varphi_{b} \rrbracket_{\eta}$ to be analyzed, where $b \in\{0,1\}$. Let $\widehat{T}$ be the answer of $\mathcal{A}$ when given the restriction of $\phi$ 
to $\operatorname{dom}(\varphi)$. $\mathcal{B}$ returns 0 if $x \phi={ }_{A_{\eta}} \llbracket \mathrm{h}_{s} \rrbracket_{A_{\eta}}(\widehat{T}, y \phi)$, and 1 otherwise. By definition, the advantage of $\mathcal{B}$ is

$$
\begin{aligned}
\mathbb{P}\left[\phi \stackrel{R}{\longleftarrow} \llbracket \varphi_{0} \rrbracket_{\eta}: \mathcal{B}(\eta, \phi)=0\right]-\mathbb{P}\left[\phi \stackrel{R}{\longleftarrow} \llbracket \varphi_{1} \rrbracket_{\eta}: \mathcal{B}(\eta, \phi)=0\right] \\
=\quad \mathbb{P}\left[\phi \stackrel{R}{\longleftarrow} \llbracket \varphi_{0} \rrbracket_{\eta} ; \widehat{T} \stackrel{R}{\longleftarrow} \mathcal{A}\left(\left.\phi\right|_{\operatorname{dom}(\varphi)}\right): x \phi={ }_{A_{\eta}} \llbracket \mathrm{h}_{s} \rrbracket_{A_{\eta}}(\widehat{T}, y \phi)\right] \\
\quad-\mathbb{P}\left[\phi \stackrel{R}{\longleftarrow} \llbracket \varphi_{1} \rrbracket_{\eta} ; \widehat{T} \stackrel{R}{\longleftarrow} \mathcal{A}\left(\left.\phi\right|_{\operatorname{dom}(\varphi)}\right): x \phi={ }_{A_{\eta}} \llbracket \mathrm{h}_{s} \rrbracket_{A_{\eta}}(\widehat{T}, y \phi)\right] \\
\left.\geq \quad \mathbb{P}\left[\phi, e \stackrel{R}{\longleftarrow} \llbracket \varphi_{0}, T \rrbracket_{\eta} ; \widehat{T} \stackrel{R}{\longleftarrow} \mathcal{A}\left(\left.\phi\right|_{\operatorname{dom}(\varphi)}\right): \widehat{T}=e\right)\right] \\
\quad-\mathbb{P}\left[\phi \stackrel{R}{\longleftarrow} \llbracket \varphi_{1} \rrbracket_{\eta} ; \widehat{T} \stackrel{R}{\longleftarrow} \mathcal{A}\left(\left.\phi\right|_{\operatorname{dom}(\varphi)}\right): x \phi={ }_{A_{\eta}} \llbracket \mathrm{h}_{s} \rrbracket_{A_{\eta}}(\widehat{T}, y \phi)\right]
\end{aligned}
$$

In the last probability expression, observe that $x \phi$ is drawn from the distribution $\left(\stackrel{R}{\longleftarrow} \llbracket H a s h \rrbracket_{A_{\eta}}\right.$ ) independently from $\widehat{T}$ and $y \phi$. Hence, as the distribution $\left(\stackrel{R}{\longleftarrow} \llbracket H a s h \rrbracket_{A_{\eta}}\right.$ ) is collision-free, the advantage of $\mathcal{B}$ is nonnegligible; contradiction.

3. Let $T_{1}$ and $T_{2}$ be two terms of sort $s$ such that $T_{1}={ }_{E} T_{2}$. Consider the same frame as before: $\varphi=\left\{x_{1}=\mathrm{h}_{s}\left(T_{1}, k\right), x_{2}=\mathrm{h}_{s}\left(T_{2}, k\right)\right\}$. As $T_{1}={ }_{E} T_{2}$ and $\mathrm{h}_{s}$ is free, we have $\varphi \approx_{E}\left\{x_{1}=n, x_{2}=n\right\}$ where $n$ is a fresh name of sort Hash (Proposition 19 of Appendix A). By assumption this entails that $\llbracket \varphi \rrbracket \approx \llbracket\left\{x_{1}=n, x_{2}=n\right\} \rrbracket$ thus

$$
\mathbb{P}\left[e_{1}^{\prime}, e_{2}^{\prime} \stackrel{R}{\longleftarrow} \llbracket \mathrm{h}_{s}\left(T_{1}, k\right), \mathrm{h}_{s}\left(T_{2}, k\right) \rrbracket_{A_{\eta}}: e_{1}^{\prime}={ }_{A_{\eta}} e_{2}^{\prime}\right] \geq 1-\epsilon_{\eta}
$$

where $\epsilon_{\eta}$ is a negligible function. As the implementation of $\mathrm{h}_{s}$ is collisionresistant, we deduce that

$$
\mathbb{P}\left[e_{1}, e_{2} \stackrel{R}{\longleftarrow} \llbracket T_{1}, T_{2} \rrbracket_{A_{\eta}}: e_{1} \neq \neq_{A_{\eta}} e_{2}\right]
$$

is negligible. Other properties follow from Proposition 1.

\section{2. $\approx_{E}$-soundness implies classical assumptions on groups}

In this section we present some interesting consequences of $\approx_{E \text {-soundness. }}$ Inspired by the work of Hohenberger and Rivest on pseudo-freeness [33, 34], we prove that several standard cryptographic assumptions on groups are implied by the soundness of static equivalence. We concentrate on abelian groups as these are more relevant for cryptographic applications. We believe that similar techniques would apply for non-commutative groups as well.

We model an abelian group $G$ with exponents taken over a commutative ring $A$ by an abstract algebra over the following signature:

$$
\begin{aligned}
& \text { * } \quad: \quad G \times G \rightarrow G \quad-\quad: \quad A \rightarrow A \\
& 1_{G}: G \quad \text {. } \quad: A \times A \rightarrow A \\
& +\quad: A \times A \rightarrow A \quad 1_{A}: A \\
& 0: A \quad \exp : G \times A \rightarrow G
\end{aligned}
$$


We use the infix notation for the operators $*, \cdot,+$, and write $g^{a}$ to denote $\exp (g, a)$. Note that the inverse operation on $G$ is represented here by $g \mapsto$ $\exp \left(g,-\left(1_{A}\right)\right)=g^{-\left(1_{A}\right)}$. We consider the equational theory $E_{\mathrm{G}}$ generated by the following equations (where $x, y, z$ are variables of sort $G$, and $u, v, w$ variables of sort $A$ ):

$$
\begin{aligned}
u+v & =v+u \\
u+(v+w) & =(u+v)+w \\
u+0_{A} & =u \\
u+(-u) & =0_{A} \\
u \cdot v & =v \cdot u \\
u \cdot(v \cdot w) & =(u \cdot v) \cdot w \\
u \cdot 1_{A} & =u \\
(u+v) \cdot w & =u \cdot w+v \cdot w
\end{aligned}
$$

$$
\begin{aligned}
x * y & =y * x \\
x *(y * z) & =(x * y) * z \\
x * 1_{G} & =x \\
\left(x^{u}\right)^{v} & =x^{(u \cdot v)} \\
x^{u} * x^{v} & =x^{u+v} \\
x^{1_{A}} & =x \\
x^{0_{A}} & =1_{G} \\
(x * y)^{u} & =x^{u} * y^{u}
\end{aligned}
$$

We now recall several classical problems on groups. For cryptographic applications, it is desirable that these problems be hard, that is, not feasible by any probabilistic polynomial-time adversary:

- discrete logarithm (DL) problem: given $g$ and $g^{\prime}$, find $a$, such that $g^{a}=g^{\prime}$;

- computational Diffie-Hellman (CDH) problem: given $g, g^{a}$ and $g^{b}$, find $g^{a b}$

- decisional Diffie-Hellman (DDH) problem: given $g, g^{a}$ and $g^{b}$, distinguish $g^{a b}$ from a random element $g^{c}$;

- RSA problem: given elements $a$ and $g^{a}$, find $g$.

A more detailed presentation of these hard problems can be found in [35].

Assume a family of computational algebras $\left(A_{\eta}\right)$ over the signature above such that $\left(A_{\eta}\right)$ is $\approx_{E_{\mathrm{G}}}$-sound, at least for some subset of well-formed frames $W F$. Consider the two frames

$$
\begin{gathered}
\varphi_{1}=\nu g, a, b \cdot\left\{x_{1}=g, x_{2}=g^{a}, x_{3}=g^{b}, x_{4}=g^{a \cdot b}\right\} \text { and } \\
\varphi_{2}=\nu g, a, b, c .\left\{x_{1}=g, x_{2}=g^{a}, x_{3}=g^{b}, x_{4}=g^{c}\right\} .
\end{gathered}
$$

and assume that $\varphi_{1}, \varphi_{2} \in W F$. Then no probabilistic polynomial-time adversary $\mathcal{A}$ can solve the DDH problem in $\left(A_{\eta}\right)$ with non-negligible probability.

Indeed, as suggested in [7], the question of (computationally) distinguishing these two frames exactly encodes the DDH problem. Given the equational theory $E_{\mathrm{G}}$, we prove the formal equivalence $\varphi_{1} \approx_{E_{\mathrm{G}}} \varphi_{2}$ (Lemma 21 of Appendix B). Thus, by $\approx_{E_{\mathrm{G}}}$-soundness, the DDH problem is hard in $\left(A_{\eta}\right)$.

Clearly, if one can solve the DL problem, one can also solve the CDH problem, which itself allows us to solve the DDH problem. Therefore, the hardness of DDH implies the hardness of the two other problems.

In a similar way, we see that $\approx_{E_{G}}$-soundness on an augmented signature implies the hardness of RSA. Instead of directly encoding the RSA problem, we introduce a slightly weaker decision problem, whose hardness implies the 
hardness of RSA. The encoding of this problem requires the extension of the signature by a unary function symbol $\mathrm{h}: G \rightarrow$ Hash, adding no equation to the theory. Consider the two frames

$$
\begin{gathered}
\varphi_{3}=\nu g, a \cdot\left\{x_{1}=g^{a}, x_{2}=a, x_{3}=\mathrm{h}(g)\right\} \text { and } \\
\varphi_{4}=\nu g, a, h \cdot\left\{x_{1}=g^{a}, x_{2}=a, x_{3}=h\right\} .
\end{gathered}
$$

We prove that $\varphi_{3} \approx_{E_{\mathrm{G}}} \varphi_{4}$ in Lemma 22 of Appendix B. As above, if an implementation $\left(A_{\eta}\right)$ is $\approx_{E_{\mathrm{G}}}$-sound of for some subset of well-formed frames $W F$ including $\varphi_{3}$ and $\varphi_{4}$, then the RSA problem cannot be efficiently solved in $\left(A_{\eta}\right)$. Indeed, any adversary $\mathcal{A}$ to the RSA-problem can be turned to an (equally efficient) adversary against $\left(\llbracket \varphi_{3} \rrbracket_{A_{\eta}}\right) \approx\left(\llbracket \varphi_{4} \rrbracket_{A_{\eta}}\right)$ simply as follows: given a sample $\left\{x_{1}=e_{1}, x_{2}=e_{2}, x_{3}=e_{3}\right\}$ from either side, let $e$ be the result of $\mathcal{A}$ applied on $\eta, e_{1}$ and $e_{2}$; return 1 ("left-hand side") if $\llbracket h \rrbracket_{A_{\eta}}(e)$ equals to $e_{3}, 0$ otherwise.

An interesting open question is whether $\approx_{E_{\mathrm{G}}}$-soundness implies or is implied by Rivest's notion of pseudo-free groups [34], or equivalently [36], the strong RSA property. We conjecture that the two notions are in fact incomparable. Indeed, on the one hand, our notion implies the hardness of DDH, which remains an open question for strong RSA. On the other hand, pseudo-freeness and strong RSA deal with a form of adaptive attackers while our model is purely nonadaptive.

\section{A sufficient (and often necessary) criterion for $\approx_{E^{-S o u n d n e s s}}$}

We now present useful results for proving $\approx_{E}$-soundness properties in gen-

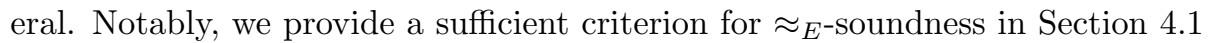
and prove it necessary under additional assumptions in Section 4.2.

\subsection{Ideal semantics and $\approx_{E}$-soundness criterion}

Given an implementation of the primitives, we have defined in Section 2.3 the concrete semantics $\llbracket \varphi \rrbracket_{A_{\eta}}$ associated to every frame $\varphi$. We now define the ideal semantics of a frame $\varphi$, intuitively as the conditional distribution over all the concrete values (in the appropriate space) that pass every formal test satisfied by $\varphi$.

Specifically, for every frame $\varphi$, we define the tests of $\varphi$ to be

$$
\operatorname{test}(\varphi)=\{(M, N) \mid \operatorname{var}(M, N) \subseteq \operatorname{dom}(\varphi), \operatorname{names}(M, N) \cap \operatorname{names}(\varphi)=\emptyset\} .
$$

We let $\operatorname{eq}_{E}(\varphi)$ be the set of tests that are true in $\varphi$ :

$$
\operatorname{eq}_{E}(\varphi)=\left\{(M, N) \in \operatorname{test}(\varphi) \mid M \varphi={ }_{E} N \varphi\right\}
$$

Note that, by definition, $\varphi \approx_{E} \varphi^{\prime}$ iff $\operatorname{eq}_{E}(\varphi) \cap \operatorname{test}\left(\varphi^{\prime}\right)=\operatorname{eq}_{E}\left(\varphi^{\prime}\right) \cap \operatorname{test}(\varphi)$.

Let $\left(A_{\eta}\right)$ be a family of computational algebras, $\varphi=\left\{x_{1}=T_{1}, \ldots, x_{n}=T_{n}\right\}$ be a frame, and $s_{i}$ be the sort of $x_{i}$. We define the set of eligible, well-formed values for $\varphi$ by

$$
\operatorname{Val}_{A_{\eta}}(\varphi)=\left\{\left\{x_{1}=e_{1}, \ldots, x_{n}=e_{n}\right\} \mid\left(e_{1}, \ldots, e_{n}\right) \in \llbracket s_{1} \rrbracket_{A_{\eta}} \times \cdots \times \llbracket s_{n} \rrbracket A_{\eta}\right\}
$$


and write $\phi \stackrel{R}{\longleftarrow} \llbracket \varphi \rrbracket_{A_{\eta}}^{\text {val }}$ for the process of drawing a random value $\phi=\left\{x_{1}=\right.$ $\left.e_{1}, \ldots, x_{n}=e_{n}\right\}$ from $\operatorname{Val}_{A_{\eta}}(\varphi)$ using the drawings $e_{i} \stackrel{R}{\longleftarrow} \llbracket s_{i} \rrbracket_{A_{\eta}}$ in the natural way.

Consider the following subset of concrete frames, intuitively, that pass all the valid tests of $\varphi$ :

$$
\begin{aligned}
\operatorname{Val}_{A_{\eta}}^{\prime}(\varphi)=\left\{\phi \in \operatorname{Val}_{A_{\eta}}(\varphi) \mid\right. & \forall(M, N) \in \mathrm{eq}_{E}(\varphi), \\
& \left.\mathbb{P}\left[u, v \stackrel{R}{\longleftarrow} \llbracket M, N \rrbracket_{A_{\eta},\left\{x_{1}=e_{1}, \ldots, x_{n}=e_{n}\right\}}: u=v\right]=1\right\}
\end{aligned}
$$

Note that, provided that $\left(A_{\eta}\right)$ is unconditionally $={ }_{E}$-sound, $\operatorname{Val}_{A_{\eta}}^{\prime}(\varphi)$ is nonempty as it contains at least the values given by the usual semantics of $\varphi$.

Definition 3 (Ideal semantics). Let $\left(A_{\eta}\right)$ be an unconditionally $={ }_{E}$-sound family of computational algebras and $\varphi$ be a frame. The ideal semantics of $\varphi$ is the family of the distributions $\llbracket \varphi \rrbracket_{A_{\eta}}^{\text {ideal }}$ obtained by conditionning each distribution $\llbracket \varphi \rrbracket_{A_{\eta}}^{\mathrm{val}}$ to the set of values $\operatorname{Val}_{A_{\eta}}^{\prime}(\varphi)$. In other words, the probability to draw $\phi \in \operatorname{Val}_{A_{\eta}}(\varphi)$ is

$$
\mathbb{P}\left[\phi \leftarrow \llbracket \varphi \rrbracket_{A_{\eta}}^{\text {ideal }}\right]= \begin{cases}0 & \text { if } \phi \notin \mathrm{Val}_{A_{\eta}}^{\prime}(\varphi) \\ \frac{1}{V} \mathbb{P}\left[\phi \stackrel{R}{\longleftarrow} \llbracket \varphi \rrbracket_{A_{\eta}}^{\text {val }}\right] & \text { otherwise }\end{cases}
$$

where $V=\mathbb{P}\left[\phi_{0} \stackrel{R}{\longleftarrow} \llbracket \varphi \rrbracket_{A_{\eta}}^{\mathrm{val}}: \phi_{0} \in \operatorname{Val}^{\prime}(\varphi)\right]$

We say that $\left(A_{\eta}\right)$ has uniform distributions if and only if for every $\eta$ and every sort $s, \llbracket s \rrbracket_{A_{\eta}}$ is a finite set, $=_{A_{\eta}, s}$ is the usual equality, and the distribution associated to $s$ by $A_{\eta}$ is the uniform one over $\llbracket s \rrbracket_{A_{\eta}}$.

By classical property of conditional probabilities, we note that in the case of uniform distributions, the ideal semantics of a frame $\varphi$ coincides with the family of uniform distributions over the (finite, non-empty) sets $\operatorname{Val}_{A_{\eta}}^{\prime}(\varphi)$.

For instance, let $\varphi=\nu n_{1}, n_{2} \cdot\left\{x_{1}=n_{1}, x_{2}=n_{2}\right\}$ with $n_{1}$ and $n_{2}$ of sort $s$. Then, given that $E$ is stable by substitution of names, we have that $\operatorname{eq}_{E}(\varphi)=$ $\left\{(M, N) \in \operatorname{test}(\varphi) \mid M={ }_{E} N\right\}$. By unconditional $={ }_{E}$-soundness, we deduce that $\llbracket \varphi \rrbracket_{A_{\eta}}^{\text {ideal }}$ is simply the uniform distribution over $\llbracket s \rrbracket_{A_{\eta}} \times \llbracket s \rrbracket_{A_{\eta}}$.

We now state our $\approx_{E}$-Soundness criterion: intuitively, the two semantics, concrete and ideal, should be indistinguishable.

Proposition 3 ( $\approx_{E}$-soundness criterion). Let $\left(A_{\eta}\right)$ be an unconditionally $={ }_{E}$-sound family of computational algebras. Assume that for every frame $\varphi$ it holds that $\left(\llbracket \varphi \rrbracket_{A_{\eta}}\right) \approx\left(\llbracket \varphi \rrbracket_{A_{\eta}}^{\text {ideal }}\right)$. Then $\left(A_{\eta}\right)$ is $\approx_{E}$-sound.

Proof. Let $\varphi_{1} \approx_{E} \varphi_{2}$. The equality $\operatorname{eq}_{E}\left(\varphi_{1}\right) \cap \operatorname{test}\left(\varphi_{2}\right)=\operatorname{eq}_{E}\left(\varphi_{2}\right) \cap \operatorname{test}\left(\varphi_{1}\right)$ entails $\operatorname{Val}_{A_{\eta}}^{\prime}\left(\varphi_{1}\right)=\mathrm{Val}_{A_{\eta}}^{\prime}\left(\varphi_{2}\right)$, thus the distributions $\llbracket \varphi_{1} \rrbracket_{A_{\eta}}^{\text {ideal }}$ and $\llbracket \varphi_{2} \rrbracket_{A_{\eta}}^{\text {ideal }}$ are equal. We use the transitivity of the indistinguishability relation $\approx$ to conclude: $\left(\llbracket \varphi_{1} \rrbracket_{A_{\eta}}\right) \approx\left(\llbracket \varphi_{1} \rrbracket_{A_{\eta}}^{\text {ideal }}\right)=\left(\llbracket \varphi_{2} \rrbracket_{A_{\eta}}^{\text {ideal }}\right) \approx\left(\llbracket \varphi_{2} \rrbracket_{A_{\eta}}\right)$. 


\subsection{Transparent frames}

In this section we show that our soundness criterion is necessary for a general class of equational theories, called transparent theories. In those theories, each frame can be associated to an equivalent transparent frame (defined below), which is easier to analyze.

Definition 4 (Transparent frames). A frame $\varphi$ is transparent for an equational theory $E$ if each of its subterms is deducible from $\varphi$ in $E$.

Example 1. In the theory $E_{\text {enc }}$, the frame $\varphi_{1}=\left\{x_{1}=\operatorname{enc}\left(\operatorname{enc}\left(k_{4}, k_{3}\right), k_{1}\right), x_{2}=\right.$ enc $\left.\left(k_{1}, k_{2}\right), x_{3}=k_{2}\right\}$ is not transparent, as neither $k_{3}$ nor $k_{4}$ are deducible, but the frame $\overline{\varphi_{1}}=\left\{x_{1}=\operatorname{enc}\left(n_{1}, k_{1}\right), x_{2}=\operatorname{enc}\left(k_{1}, k_{2}\right), x_{3}=k_{2}\right\}$ is.

The following proposition finitely characterizes the equations verified by a transparent frame.

Proposition 4. Let $\varphi$ be a transparent frame for E. Then, $\varphi$ is of the form

$$
\varphi=\left\{x_{1}=C_{1}\left[a_{1}, \ldots, a_{m}\right], \ldots, x_{n}=C_{n}\left[a_{1}, \ldots, a_{m}\right]\right\}
$$

where $C_{1}, \ldots, C_{n}$ are (not necessarily linear) contexts such that names $\left(C_{1}, \ldots, C_{n}\right)=$ $\emptyset, C_{1}\left[a_{1}, \ldots, a_{m}\right], \ldots, C_{n}\left[a_{1}, \ldots, a_{m}\right]$ are closed and, $a_{1}, \ldots, a_{m}$ are distinct deducible names: $\varphi \vdash_{E} a_{i}$.

For each $a_{i}$, let $M_{a_{i}}$ be a term such that $\operatorname{var}\left(M_{a_{i}}\right) \subseteq\left\{x_{1}, \ldots, x_{n}\right\}$, names $\left(M_{a_{i}}\right) \cap$ $\operatorname{names}(\varphi)=\emptyset$ and $M_{a_{i}} \varphi={ }_{E} a_{i}$. Then every equation which holds in $\varphi$ is a logical consequence of $E$ and the equations $x_{j}=C_{j}\left[M_{a_{1}}, \ldots, M_{a_{m}}\right]$, written

$$
E \cup\left\{x_{j}=C_{j}\left[M_{a_{1}}, \ldots, M_{a_{m}}\right] \mid 1 \leq j \leq n\right\} \mid=\operatorname{eq}_{E}(\varphi) .
$$

By logical consequence, we refer to the usual first-order theory of equality, where the variables $x_{1}, \ldots, x_{n}$ are considered here as constants.

Proof. Let $(M, N) \in \operatorname{eq}_{E}(\varphi)$. By definition, we have $M \varphi=_{E} N \varphi$, that is, $M\left\{x_{j} \mapsto C_{j}\left[a_{1}, \ldots, a_{m}\right]\right\}_{1 \leq j \leq n}=_{E} N\left\{x_{j} \mapsto C_{j}\left[a_{1}, \ldots, a_{m}\right]\right\}_{1 \leq j \leq n}$. Since $E$ is stable by substitution of names, we obtain

$$
M\left\{x_{j} \mapsto C_{j}\left[M_{a_{1}}, \ldots, M_{a_{m}}\right]\right\}_{1 \leq j \leq n}={ }_{E} N\left\{x_{j} \mapsto C_{j}\left[M_{a_{1}}, \ldots, M_{a_{m}}\right]\right\}_{1 \leq j \leq n} .
$$

Using the equalities $x_{j}=C_{j}\left[M_{a_{1}}, \ldots, M_{a_{m}}\right]$ and by transitivity, we obtain $\left\{x_{j}=\right.$ $\left.C_{j}\left[M_{a_{1}}, \ldots, M_{a_{m}}\right] \mid 1 \leq j \leq n\right\} \cup E \models M=N$.

Another nice and useful property of transparent frames is that their concrete and ideal semantics coincide.

Proposition 5. Let $\left(A_{\eta}\right)$ be an unconditionally $={ }_{E}$-sound family of computational algebras, having uniform distributions. Let $\varphi$ be a transparent frame. The concrete and the ideal semantics of $\varphi$ yield the same family of distributions: for all $\eta, \llbracket \varphi \rrbracket_{A_{\eta}}=\llbracket \varphi \rrbracket_{A_{\eta}}^{\text {ideal }}$. 
Proof. Let $\varphi=\left\{x_{1}=C_{1}\left[a_{1}, \ldots, a_{m}\right], \ldots, x_{n}=C_{n}\left[a_{1}, \ldots, a_{m}\right]\right\}$, with $M_{i} \varphi={ }_{E}$ $a_{i}(1 \leq i \leq m)$ as above. Let $s_{i}$ be the sort of $a_{i}, s_{j}^{\prime}$ be the sort of $x_{j}$ and $\eta$ a given complexity parameter.

The usual concrete semantics of $\varphi$ consists in mapping every drawing of names from the set $\mathcal{E}=\llbracket s_{1} \rrbracket_{A_{\eta}} \times \cdots \times \llbracket s_{m} \rrbracket_{A_{\eta}}$ to a value in $\mathcal{F}=\operatorname{Val}_{A_{\eta}}(\varphi)$. Let us note $\alpha: \mathcal{E} \rightarrow \mathcal{F}$ this function, defined by:

$$
\begin{array}{r}
\alpha\left(e_{1}, \ldots, e_{m}\right)=\left\{x_{1}=\llbracket C_{1}\left[y_{1}, \ldots, y_{m}\right] \rrbracket_{\left\{y_{1}=e_{1}, \ldots, y_{m}=e_{m}\right\}}, \ldots,\right. \\
\left.x_{n}=\llbracket C_{n}\left[y_{1}, \ldots, y_{m}\right] \rrbracket_{\left\{y_{1}=e_{1}, \ldots, y_{m}=e_{m}\right\}}\right\}
\end{array}
$$

where the $y_{i}$ are fresh variables respectively of sort $s_{i}$, and we omit the subscript $A_{\eta}$ for sake of clarity.

Using the $M_{i}$, we can also define a function $\beta: \mathcal{F} \rightarrow \mathcal{E}$ :

$$
\beta(\phi)=\left(\llbracket M_{1} \rrbracket_{\phi}, \ldots, \llbracket M_{m} \rrbracket_{\phi}\right)
$$

We note that the distribution of $\llbracket M_{i} \varphi \rrbracket$ equals to that of $\llbracket M_{i} \rrbracket_{\phi}$ where $\phi \stackrel{R}{\longleftarrow}$ $\llbracket \varphi \rrbracket$, or equivalently, of $\llbracket M_{i} \rrbracket_{\alpha\left(e_{1}, \ldots, e_{n}\right)}$ where $\left(e_{1}, \ldots, e_{n}\right) \stackrel{R}{\leftarrow} \mathcal{E}$. As $M_{i} \varphi={ }_{E} a_{i}$, $\left(A_{\eta}\right)$ is unconditionally $=_{E}$-sound, and no element of $\mathcal{E}$ has probability 0 , we obtain that $\beta \circ \alpha=I d_{E}$. Thus $\alpha$ is injective and yields a bijection from $\mathcal{E}$ to its image $\mathcal{G}=\alpha(\mathcal{E})$. By assumption, $\mathcal{E}$ is equipped with the uniform distribution, therefore the concrete semantics of $\varphi$ is the uniform distribution on $\mathcal{G}$.

Moreover $\mathcal{G}$ satisfies:

$$
\begin{aligned}
\mathcal{G} & =\{\phi \in \mathcal{F} \mid \alpha(\beta(\phi))=\phi\} \\
& =\left\{\phi \in \mathcal{F} \mid \forall j, \llbracket C_{j}\left[y_{1}, \ldots, y_{m}\right] \rrbracket_{\left\{y_{1}=\llbracket M_{1} \rrbracket_{\phi}, \ldots, y_{m}=\llbracket M_{i} \rrbracket_{\phi}\right\}}=\llbracket x_{j} \rrbracket_{\phi}\right\} \\
& =\left\{\phi \in \mathcal{F} \mid \forall j, \llbracket C_{j}\left[M_{1}, \ldots, M_{m}\right] \rrbracket_{\phi}=\llbracket x_{j} \rrbracket_{\phi}\right\}
\end{aligned}
$$

As $\varphi$ is transparent, by Proposition $4, \operatorname{eq}_{E}(\varphi)$ is implied by the equations $C_{j}\left[M_{1}, \ldots, M_{m}\right]=x_{j}$ and $E$. By unconditional $={ }_{E}$-soundness, we deduce that the values in $\mathcal{G}$ pass all the tests in $\operatorname{eq}_{E}(\varphi)$; in other words, $\mathcal{G} \subseteq \operatorname{Val}_{A_{\eta}}^{\prime}(\varphi)$. Conversely, every element of $\operatorname{Val}_{A_{\eta}}^{\prime}(\varphi)$ is trivially in $\mathcal{G}$; therefore $\mathcal{G}=\operatorname{Val}_{A_{\eta}}^{\prime \prime}(\varphi)$. Since $\mathcal{F}$ is equipped with uniform distribution, we obtain that the ideal semantics of $\varphi$ coincides with the uniform distribution on $\mathcal{G}$, and therefore with its concrete semantics.

A noticeable consequence of Proposition 5 is that, in the case of uniform distributions, two statically-equivalent transparent frames are always indistinguishable. (The argument is similar to that of Proposition 3.) This motivates the following definition, for the purpose of studying $\approx_{E}$-soundness or a converse to Proposition 3.

Definition 5. An equational theory $E$ is transparent if and only if for every frame $\varphi$, there exists a (not necessarily unique) transparent frame $\bar{\varphi}$ such that $\varphi \approx_{E} \bar{\varphi}$. 
Transparent frames and theories are related to the notion of patterns introduced by Abadi and Rogaway [4] and used in subsequent work [13, 12] so as to define computationally sound formal equivalences. There, messages are first mapped to patterns by replacing non-deducible subterms with boxes $\square$. By definition, two messages are then equivalent if and only if they yield the same pattern (up to renaming of names). For example, if $\{M\}_{K}$ denotes the probabilistic encryption of $M$ by a key $K$, the message $\left(\left\{\left\{K_{4}\right\}_{K_{3}}\right\}_{K_{1}},\left\{K_{1}\right\}_{K_{2}}, K_{2}\right)$ is mapped to the pattern $\left(\{\square\}_{K_{1}},\left\{K_{1}\right\}_{K_{2}}, K_{2}\right)$. (Compare with example 1 where we have $\varphi_{1} \approx_{E_{\text {enc }}} \overline{\varphi_{1}}$.)

However, the notion of transparent frames is defined for any equational theory. Also, it might be the case that a frame corresponds to several transparent frames. For example, consider the theory of the exclusive OR (given in Section 5.1) and the frame:

$$
\varphi=\left\{x_{1}=n_{1} \oplus n_{2}, x_{2}=n_{2} \oplus n_{3}, x_{3}=n_{1} \oplus n_{3}\right\} .
$$

There are several transparent frames equivalent to $\varphi$, for instance $\left\{x_{1}=n_{1} \oplus\right.$ $\left.n_{2}, x_{2}=n_{1}, x_{3}=n_{2}\right\},\left\{x_{1}=n_{1}, x_{2}=n_{1} \oplus n_{2}, x_{3}=n_{2}\right\}$ and $\left\{x_{1}=n_{1}, x_{2}=\right.$ $\left.n_{2}, x_{3}=n_{1} \oplus n_{2}\right\}$.

We believe that the notion of transparent frames is relevant in many theories useful in cryptography. As a matter of fact, the two theories of exclusive OR and ciphers considered in Section 5 are transparent. However, the notion of transparent frames does not subsume that of patterns, defined by Abadi and Rogaway. In particular, for the theory of probabilistic symmetric encryption, that is,

$$
E_{\mathrm{senc}}=\left\{\operatorname{sdec}(\operatorname{senc}(x, y, z), y)=x, \quad \operatorname{sdec} \_\operatorname{success}(\operatorname{senc}(x, y, z), y)=\mathrm{ok}\right\}
$$

it is unclear how to associate an equivalent transparent frame to the frame $\nu n, k, r .\{x=\operatorname{senc}(n, k, r), y=k\}$, although it is arguably a pattern in the sense of Abadi and Rogaway (once cast into our syntax). The reason is that the random coin $r$ is not deducible, but the term $\operatorname{senc}(n, k, r)$ cannot be replaced with a fresh name because of the visible equation $\operatorname{sdec} \_\operatorname{success}(x, y)=\mathrm{ok}$. We might exclude $r$ from being a subterm by modifying the notion of subterms (for example, in Abadi and Rogaway's work, the random factor does not appear explicitely in terms). However, this would undermine the properties of transparent frames mentioned above. Thus, we regard the notions of patterns and transparent frames as complementary.

Note that we have proved en passant that $\approx_{E}$ is decidable for transparent theories $E$ for which $=_{E}$ is decidable, provided that the reduction to equivalent transparent frames is effective. Indeed, given two frames $\varphi_{1}$ and $\varphi_{2}$, we associate to each of them one of its statically equivalent transparent frame $\overline{\varphi_{1}}$ and $\overline{\varphi_{2}}$, respectively. It is then straightforward to check whether $\overline{\varphi_{1}}$ and $\overline{\varphi_{2}}$ are equivalent using the finite characterization of $\mathrm{eq}_{E}\left(\overline{\varphi_{i}}\right)$ by Proposition 4.

Finally, we establish a completeness result for our soundness criterion in the cases of transparent theories. 
Theorem 6. Assume a transparent theory E. Let $\left(A_{\eta}\right)$ be a family of computational algebras such that $\left(A_{\eta}\right)$ has uniform distributions, is $\approx_{E}$-sound and unconditionally $={ }_{E}$-sound. Then the soundness criterion of Proposition 3 is satisfied: for every frame $\varphi,\left(\llbracket \varphi \rrbracket_{A_{\eta}}\right) \approx\left(\llbracket \varphi \rrbracket_{A_{\eta}}^{\text {ideal }}\right)$.

Proof. Since $E$ is transparent, there exists a transparent frame $\bar{\varphi}$ such that $\varphi \approx_{E} \bar{\varphi}$. By $\approx_{E}$-soundness, we deduce $\left(\llbracket \varphi \rrbracket_{A_{\eta}}\right) \approx\left(\llbracket \bar{\varphi} \rrbracket_{A_{\eta}}\right)$. By Proposition 5, we have that $\left(\llbracket \bar{\varphi} \rrbracket A_{A_{\eta}}\right)=\left(\llbracket \bar{\varphi} \rrbracket_{A_{\eta}}^{\text {ideal }}\right)$. Altogether, we conclude that $\left(\llbracket \varphi \rrbracket_{A_{\eta}}\right) \approx$ $\left(\llbracket \varphi \rrbracket_{A_{\eta}}^{\text {ideal }}\right)$ since $\varphi \approx_{E} \bar{\varphi}$ implies $\left(\llbracket \bar{\varphi} \rrbracket_{A_{\eta}}^{\text {ideal }}\right)=\left(\llbracket \varphi \rrbracket_{A_{\eta}}^{\text {ideal }}\right)$ as before.

\section{Examples}

We now apply the framework of Sections 3 and 4 to establish two $\approx_{E^{-}}$ soundness results, concerning the theory of exclusive OR and that of ciphers and lists.

\subsection{Exclusive OR}

We study the soundness and faithfulness problems for the natural theory and implementation of the exclusive OR (XOR), together with constants and (pure) random numbers.

The formal model consists of a single sort Data, an infinite number of names, the infix symbol $\oplus:$ Data $\times$ Data $\rightarrow$ Data and two constants $0,1:$ Data. Terms are equipped with the equational theory $E_{\oplus}$ generated by:

$$
\begin{aligned}
(x \oplus y) \oplus z & =x \oplus(y \oplus z) & x \oplus x & =0 \\
x \oplus y & =y \oplus x & & x \oplus 0=x
\end{aligned}
$$

As an implementation, we define the computational algebras $A_{\eta}, \eta \geq 0$ :

- the concrete domain $\llbracket D a t a \rrbracket_{A_{\eta}}$ is the set of bit-strings of length $\eta,\{0,1\}^{\eta}$, equipped with the uniform distribution;

- $\oplus$ is interpreted by the usual XOR function over $\{0,1\}^{\eta}$;

- $\llbracket 0 \rrbracket_{A_{\eta}}=0^{\eta}$ and $\llbracket 1 \rrbracket_{A_{\eta}}=1^{\eta}$.

In this setting, statically equivalent frames enjoy an algebraic characterization. Let $A C$ be the equational theory corresponding to the two left-hand equations for associativity and commutativity. We use the other two equations as a rewriting system $\mathcal{R}_{\oplus}$

$$
\begin{aligned}
& x \oplus x \rightarrow 0 \\
& x \oplus 0 \rightarrow x
\end{aligned}
$$

where we allow arbitrary $A C$-manipulations before and after each rewriting step. It is easy to show that $\mathcal{R}_{\oplus}$ is $A C$-convergent. Specifically, a term $T$ is in $\mathcal{R}_{\oplus} / A C$-normal form (or simply normal form in the following) if and only if each name, variable and constant 1 occur at most once in $T$, and 0 does not occur in $T$ unless $T=0$. 
Let $a_{1}, \ldots, a_{n}$ be distinct names. Using the rewriting system $\mathcal{R}_{\oplus} / A C$, every closed term $T$ with names $(T) \subseteq\left\{a_{1}, \ldots, a_{n}\right\}$ can be written $T={ }_{E_{\oplus}}$ $\beta_{0} \oplus \bigoplus_{j=1}^{n} \beta_{j} a_{j}$ where $\beta_{j} \in\{0,1\}$, the $a_{j}$ are mutually distinct, and we use the convention $0 a_{j}=0$ and $1 a_{j}=a_{j}$. In the following, we see $\{0,1\}$ as the two-element field $\mathbb{F}_{2}$; thus terms modulo $=_{E_{\oplus}}$ form a $\mathbb{F}_{2}$-vector space.

Similarly a frame $\varphi$ with $\operatorname{names}(\varphi) \subseteq\left\{a_{1}, \ldots, a_{n}\right\}$ is written

$$
\varphi=E_{\oplus}\left\{x_{1}=\alpha_{1,0} \oplus \bigoplus_{j=1}^{n} \alpha_{1, j} a_{j}, \ldots, x_{m}=\alpha_{m, 0} \oplus \bigoplus_{j=1}^{n} \alpha_{m, j} a_{j}\right\}
$$

where $\alpha_{i, j} \in \mathbb{F}_{2}$. Let us group the coefficients into a $(m+1) \times(n+1)$-matrix $\alpha=\left(\alpha_{i, j}\right)$ over $\mathbb{F}_{2}$. Then, $\varphi$ is described by the formal relation

$$
\left(\begin{array}{c}
1 \\
x_{1} \\
\vdots \\
x_{m}
\end{array}\right)=\underbrace{\left(\begin{array}{cccc}
1 & 0 & \ldots & 0 \\
\alpha_{1,0} & \alpha_{1,1} & \ldots & \alpha_{1, n} \\
\vdots & & & \vdots \\
\alpha_{m, 0} & \alpha_{m, 1} & \ldots & \alpha_{m, n}
\end{array}\right)}_{\alpha} \cdot\left(\begin{array}{c}
1 \\
a_{1} \\
\vdots \\
a_{n}
\end{array}\right)
$$

We now characterize the set $\operatorname{eq}_{E_{\oplus}}(\varphi)$ of equations valid in $\varphi$. Let $M$ and $N$ be two terms such that $\operatorname{var}(M, N) \subseteq \operatorname{dom}(\phi), \operatorname{names}(M, N) \cap \operatorname{names}(\varphi)=\emptyset$. First note that $M \varphi=E_{E_{\oplus}} N \varphi$ if and only if $(M \oplus N) \varphi==_{E_{\oplus}} 0$. Therefore we only study the case where $N=0$.

Assume $M$ in normal form. $M \varphi={ }_{E_{\oplus}} 0$ and names $(M) \cap \operatorname{names}(\varphi)=\emptyset$ implies names $(M)=\emptyset$. Let $M={ }_{A C} \beta_{0} \oplus \bigoplus_{i=1}^{m} \beta_{i} x_{i}$. The condition $M \varphi=E_{\oplus} 0$ is equivalent to the vectorial equation

$$
\left(\beta_{0}, \ldots, \beta_{m}\right) \cdot \alpha=0
$$

that is, $\left(\beta_{0}, \ldots, \beta_{m}\right)$ belongs to the co-kernel of $\alpha$, noted $\operatorname{coker}(\alpha)$.

Finally let $\varphi$ and $\varphi^{\prime}$ be two frames with names $\left(\varphi, \varphi^{\prime}\right) \subseteq\left\{a_{1}, \ldots, a_{n}\right\}$ and $\operatorname{dom}(\varphi)=\operatorname{dom}\left(\varphi^{\prime}\right)=\left\{x_{1}, \ldots, x_{m}\right\}$. Let $\alpha$ and $\alpha^{\prime}$ be the two corresponding $(m+1) \times(n+1)$-matrices defined as above. From the previous discussion, we deduce that

$$
\varphi \approx_{E_{\oplus}} \varphi^{\prime} \Leftrightarrow \operatorname{coker}(\alpha)=\operatorname{coker}\left(\alpha^{\prime}\right)
$$

that is, if we write $\operatorname{im}(\alpha)=\{\alpha \cdot \gamma\}$ the image of $\alpha$, we have by duality

$$
\varphi \approx_{E_{\oplus}} \varphi^{\prime} \Leftrightarrow \operatorname{im}(\alpha)=\operatorname{im}\left(\alpha^{\prime}\right) .
$$

This characterization is the key point of our main result for the theory of XOR.

Theorem 7. The implementation of XOR for the considered signature, $\left(A_{\eta}\right)$, is unconditionally $=_{E_{\oplus}}$, $\approx_{E_{\oplus}}$ and $\forall_{E_{\oplus}}$-sound. It is also $=_{E_{\oplus}}{ }^{-}, \approx_{E_{\oplus}}$ and $\forall E_{\oplus}-$ faithful. 
Proof. The unconditional $=_{E_{\oplus}}$-soundness is clear, hence the $\nvdash_{E_{\oplus}}$-faithfulness (Proposition 1).

Let us show that $\left(A_{\eta}\right)$ is $=_{E_{\oplus}}$-faithful. Assume that $T_{1}$ and $T_{2}$ are two terms such that $T_{1} \neq_{E_{\oplus}} T_{2}$. This is equivalent to $T_{1} \oplus T_{2} \neq_{E_{\oplus}} 0$. Thus it is sufficient to consider the case where $T \neq 0$ is a closed term in normal form. The semantics of $T$ is either the constant $1^{\eta}$ (if $T=1$ ) or the uniform distribution (if $T \neq 1$ ) on $\{0,1\}^{\eta}$. Thus $\mathbb{P}\left[\llbracket T \rrbracket_{A_{\eta}}=0\right]$ is negligible. Hence the $=_{E_{\oplus}}$-faithfulness holds and by proposition 1 , so does the $\approx_{E_{\oplus}}$-faithfulness.

We now address the unconditional $\approx_{E_{\oplus}}$-soundness. Let $\varphi$ be a frame, and $\alpha=\left(\alpha_{i, j}\right)$ its $(m+1) \times(n+1)$-matrix associated as before. Let us see $\alpha$ as a $\mathbb{F}_{2}$-linear function from $\left(\mathbb{F}_{2}\right)^{n+1}$ to $\left(\mathbb{F}_{2}\right)^{m+1}$.

For simplicity, let us fix the order of variables in $\operatorname{dom}(\varphi)$ and assimilate the possible concrete values of $\varphi, \operatorname{Val}_{A_{\eta}}(\varphi)$, to the set $\mathcal{F}=\left\{1^{\eta}\right\} \times\left(\mathbb{F}_{2}\right)^{m \eta}$ where the first $\eta$ 1-bits are added for technical reasons.

The usual concrete semantics of $\varphi$ consists in drawing a random vector uniformly from $\mathcal{E}=\left\{1^{\eta}\right\} \times\left(\mathbb{F}_{2}\right)^{n \eta}$ for the value of names, and then applying a $\mathbb{F}_{2}$-linear function $\widehat{\alpha}:\left(\mathbb{F}_{2}\right)^{(n+1) \eta} \rightarrow\left(\mathbb{F}_{2}\right)^{(m+1) \eta}$ to it. Specifically, if we see $\left(\mathbb{F}_{2}\right)^{(n+1) \eta}$ as $\underbrace{\mathbb{F}_{2}^{\eta} \times \ldots \times \mathbb{F}_{2} \eta}_{n+1}$ and similarly for $\left(\mathbb{F}_{2}\right)^{(m+1) \eta}$, the function $\widehat{\alpha}$ is defined by

$$
\widehat{\alpha}\left(f_{0}, \ldots, f_{n}\right)=\left(\bigoplus_{j=0}^{n} \alpha_{0, j} f_{j}, \ldots, \bigoplus_{j=0}^{n} \alpha_{m, j} f_{j}\right)
$$

Since $\widehat{\alpha}$ is linear, all the inverse images $\widehat{\alpha}^{-1}(\{x\}), x \in \operatorname{im}(\widehat{\alpha})$, have the same cardinal. Hence, the concrete semantics of $\varphi$ is also the uniform distribution over $\widehat{\alpha}(\mathcal{E})=\operatorname{im}(\widehat{\alpha}) \cap \mathcal{F}$.

Assume a second frame $\varphi^{\prime}$ such that $\varphi \approx_{E_{\oplus}} \varphi^{\prime}$. Define $\alpha^{\prime}$ and $\widehat{\alpha^{\prime}}$ similarly as above. By equation 1 , we have $\operatorname{im}(\alpha)=\operatorname{im}\left(\alpha^{\prime}\right)$.

Now, if we see $\left(\mathbb{F}_{2}\right)^{(m+1) \eta}$ as $\underbrace{\mathbb{F}_{2}{ }^{m+1} \times \ldots \times \mathbb{F}_{2}{ }^{m+1}}_{\eta}$, we may write $\widehat{\alpha}=$ $\underbrace{\alpha \times \ldots \times \alpha}_{\eta}$ and similarly for $\alpha^{\prime}$. Thus,

$$
\operatorname{im}(\widehat{\alpha})=\underbrace{\operatorname{im}(\alpha) \times \ldots \times \operatorname{im}(\alpha)}_{\eta}=\underbrace{\operatorname{im}\left(\alpha^{\prime}\right) \times \ldots \times \operatorname{im}\left(\alpha^{\prime}\right)}_{\eta}=\operatorname{im}\left(\widehat{\alpha^{\prime}}\right)
$$

which implies that $\varphi$ and $\varphi^{\prime}$ have the same concrete semantics. Thus $E_{\oplus}$ is unconditionally $\approx_{E_{\oplus}}$-sound.

Last, we prove the unconditional $\nvdash_{E_{\oplus}}$-soundness. Let $\varphi$ be a frame and $T$ a term, both in normal form, such that $\varphi \nvdash_{E_{\oplus}} T$ and names $(T) \subseteq \operatorname{names}(\varphi)=$ $\left\{a_{1}, \ldots, a_{n}\right\}$. Let $\alpha$ be associated to $\varphi$ as before and $T={ }_{A C} \beta_{0} \oplus \bigoplus_{j=1}^{n} \beta_{j} a_{j}$.

Let $\gamma$ be the $(m+2) \times(n+1)$-matrix obtained by augmenting $\alpha$ with a last 
row equal to $\beta=\left(\beta_{0}, \ldots, \beta_{n}\right)$ :

$$
\gamma=\left(\begin{array}{cccc}
1 & 0 & \ldots & 0 \\
\alpha_{1,0} & \alpha_{1,1} & \ldots & \alpha_{1, n} \\
\vdots & & & \vdots \\
\alpha_{m, 0} & \alpha_{m, 1} & \ldots & \alpha_{m, n} \\
\beta_{0} & \beta_{1} & \ldots & \beta_{n_{0}}
\end{array}\right)
$$

Since $\varphi \forall_{E_{\oplus}} T$, in particular there exists no $M$ in normal form such that names $(M)=\emptyset$ and $M \varphi={ }_{E_{\oplus}} T$. In other words, $\beta$ is linearly independent from the other rows in the matrix $\gamma$ above.

In particular, it is independent from the first row $(1,0, \ldots, 0)$, that is, there exists $j \geq 1$ such that $\beta_{j} \neq 0$. We deduce that the distribution $\left(\stackrel{R}{\longleftarrow} \llbracket T \rrbracket_{A_{\eta}}\right)$ is the uniform one over $\{0,1\}^{\eta}$, thus it is collision-free.

As for the first condition of unconditional $\forall_{E}$-soundness, by a similar reasoning as before, we have that the concrete semantics of $(\varphi, T)$ is the uniform distribution over the image of $\mathcal{E}=\left\{1^{\eta}\right\} \times\left(\mathbb{F}_{2}\right)^{n \eta}$ by $\widehat{\gamma}$ (defined similarly as $\widehat{\alpha}$ above). Let us see $\beta$ a linear function from $\left(\mathbb{F}_{2}\right)^{n+1}$ to $\mathbb{F}_{2}$ and define $\widehat{\beta}$ as previously. Next we prove that the image $\widehat{\gamma}(\mathcal{E})$ is the cartesian product of the two sets $\widehat{\alpha}(\mathcal{E})$ and $\widehat{\beta}(\mathcal{E})$. It follows that the drawings for $\varphi$ and $T$ are independent.

The inclusion $\widehat{\gamma}(\mathcal{E}) \subseteq \widehat{\alpha}(\mathcal{E}) \times \widehat{\beta}(\mathcal{E})$ is trivial. As $\beta$ is independent from the rows of $\alpha$, there exists a vector $u \in\left(\mathbb{F}_{2}\right)^{n+1}$ such that $\beta(u)=1$ and $\alpha(u)=0$ (otherwise $\operatorname{ker}(\beta) \supseteq \operatorname{ker}(\alpha)$ implies $\beta \in \operatorname{coim}(\beta) \subseteq \operatorname{coim}(\alpha)$ ). Let $x, y \in \mathcal{E}$. We prove that there exists $z \in \mathcal{E}$ such that $\widehat{\alpha}(z)=\widehat{\alpha}(x) \in\left(\mathbb{F}_{2}\right)^{(m+1) \eta}$ and $\widehat{\beta}(z)=\widehat{\beta}(y) \in\left(\mathbb{F}_{2}\right)^{\eta}$.

Indeed, let us see $\mathcal{E}$ as $\left(\{1\} \times\left(\mathbb{F}_{2}\right)^{n}\right)^{\eta}$. Using the corresponding bases, let $x=\left(x_{1}, \ldots, x_{\eta}\right)$ and $y=\left(y_{1}, \ldots, y_{\eta}\right)$ with $x_{i}, y_{i} \in\{1\} \times\left(\mathbb{F}_{2}\right)^{n}$. We let $z_{i}=$ $x_{i}+\left(\beta\left(y_{i}\right)-\beta\left(x_{i}\right)\right) \cdot u$ and $z=\left(z_{1}, \ldots, z_{\eta}\right)$. Thus, $\widehat{\alpha}(z)=\left(\alpha\left(z_{1}\right), \ldots, \alpha\left(z_{\eta}\right)\right)=$ $\left(\alpha\left(x_{1}\right), \ldots, \alpha\left(x_{\eta}\right)\right)=\widehat{\alpha}(x)$ and $\widehat{\beta}(z)=\left(\beta\left(z_{1}\right), \ldots, \beta\left(z_{\eta}\right)\right)=\left(\beta\left(y_{1}\right), \ldots, \beta\left(y_{\eta}\right)\right)=$ $\widehat{\beta}(y)$. Besides, $\alpha(u)=0$ implies that the first coordinate of $u$ is 0 , thus the first coordinate of each $z_{i}$ is 1 , that is, $z \in \mathcal{E}$.

We conclude this section by a proof that the $E_{\oplus}$ is transparent as announced in Section 4.

Proposition 8. The equational theory $E_{\oplus}$ is transparent.

Proof. Indeed, let $\varphi$ be frame and $\alpha$ be its associated $(m+1) \times(n+1)$-matrix as before. Let $d$ be the dimension of $\operatorname{im}(\alpha)$. There exists a $(m+1) \times d$ submatrix $\alpha^{\prime}$ of $\alpha$ such that $\alpha^{\prime}$ is injective and $\operatorname{im}\left(\alpha^{\prime}\right)=\operatorname{im}(\alpha)$ (consider a maximal independent set of columns of $\alpha$ ). As the first column of $\alpha$ is independent from the others (it starts with a 1 whereas the others start with a 0 ), we may assume without loss of generality that the first column of $\alpha^{\prime}$ is that of $\alpha$. (In particular $d \geq 1$.) 
Let $a_{1}^{\prime} \ldots a_{d-1}^{\prime}$ be distinct names. We let $\varphi^{\prime}$ be the frame associated to $\alpha^{\prime}$, described by the relation

$$
\left(\begin{array}{c}
1 \\
x_{1} \\
\vdots \\
x_{m}
\end{array}\right)=\alpha^{\prime} \cdot\left(\begin{array}{c}
1 \\
a_{1}^{\prime} \\
\vdots \\
a_{d-1}^{\prime}
\end{array}\right) .
$$

As $\operatorname{im}\left(\alpha^{\prime}\right)=\operatorname{im}(\alpha)$, we have $\varphi^{\prime} \approx_{E_{\oplus}} \varphi$. Besides, since $\alpha^{\prime}$ is injective, there exists $\alpha^{\prime \prime}$ such that $\alpha^{\prime \prime} \cdot \alpha^{\prime}$ is the identity $d \times d$-matrix. This entails that every $a_{i}^{\prime}$ is deducible from $\varphi^{\prime}$, that is, $\varphi^{\prime}$ is transparent.

\subsection{Symmetric, deterministic, length-preserving encryption and lists}

We now detail the example of symmetric, deterministic and length-preserving encryption schemes. Such schemes, also known as pseudo-random permutations or ciphers [37], are widely used in practice, the most famous examples (for fixed-length inputs) being DES and AES.

Our formal model consists of a set of sorts $\mathcal{S}=\left\{\right.$ Data, List $_{0}$, List $_{1} \ldots$ List $\left._{n} \ldots\right\}$, an infinite number of names for every sort Data and List $_{n}$, and the following symbols (for every $n \geq 0$ ):

$$
\begin{aligned}
& \text { enc }_{n}, \text { dec }_{n} \quad: \quad \text { List }_{n} \times \text { Data } \rightarrow \text { List }_{n} \quad \text { encryption, decryption } \\
& \text { cons }_{n} \quad: \quad \text { Data } \times \text { List }_{n} \rightarrow \text { List }_{n+1} \quad \text { list constructor } \\
& \text { head }_{n}: \text { List }_{n+1} \rightarrow \text { Data } \quad \text { head of a list } \\
& \text { tail }_{n}: \text { List }_{n+1} \rightarrow \text { List }_{n} \quad \text { tail of a list } \\
& \text { nil : } \text { List }_{0} \quad \text { empty list } \\
& 0,1 \text { : Data constants }
\end{aligned}
$$

We consider the equational theory $E_{\text {sym }}$ generated by the following equations (for every $n \geq 0$ and for every name $a_{0}$ of sort List $_{0}$ ):

$$
\begin{aligned}
\operatorname{dec}_{n}\left(\operatorname{enc}_{n}(x, y), y\right) & =x & \operatorname{enc}_{0}(\text { nil }, x) & =\text { nil } \\
\operatorname{enc}_{n}\left(\operatorname{dec}_{n}(x, y), y\right) & =x & \operatorname{dec}_{0}(\text { nil }, x) & =\text { nil } \\
\operatorname{head}_{n}\left(\operatorname{cons}_{n}(x, y)\right) & =x & \operatorname{tail}_{0}(x) & =\text { nil } \\
\operatorname{tail}_{n}\left(\operatorname{cons}_{n}(x, y)\right) & =y & a_{0} & =\text { nil } \\
\operatorname{cons}_{n}\left(\operatorname{head}_{n}(x), \operatorname{tail}_{n}(x)\right) & =x & &
\end{aligned}
$$

where $x, y$ are variables of the appropriate sorts in each case. The effect of the last four equations is that the sort List $_{0}$ is degenerated in $E_{\text {sym }}$, that is, all terms of sort List $_{0}$ are equal. When oriented from left to right, the equations above form a convergent rewriting system written $\mathcal{R}$.

Notice that each term has a unique sort. As the subscripts $n$ of function symbols are redundant with sorts, we tend to omit them in terms. For instance, if $k, k^{\prime}:$ Data, we may write enc(cons $\left(k\right.$, nil), $\left.k^{\prime}\right)$ instead of enc ${ }_{1}\left(\operatorname{cons}_{0}\left(k\right.\right.$, nil), $\left.k^{\prime}\right)$.

The concrete meaning of sorts and symbols is given by the computational algebras $A_{\eta}, \eta>0$, defined as follows: 
- the carrier sets are $\llbracket D a t a \rrbracket_{A_{\eta}}=\{0,1\}^{\eta}$ and $\llbracket$ List $_{n} \rrbracket_{A_{\eta}}=\{0,1\}^{n \eta}$ equipped with the uniform distribution and the usual equality relation;

- enc $_{n}, \operatorname{dec}_{n}$ are implemented by a cipher for data of size $n \eta$ and keys of size $\eta$; (we discuss the required cryptographic assumptions later);

- $\llbracket$ nil $\rrbracket_{A_{\eta}}$ is the empty bit-string, $\llbracket$ cons $_{n} \rrbracket_{A_{\eta}}$ is the usual concatenation, $\llbracket 0 \rrbracket_{A_{\eta}}=$ $0^{\eta}, \llbracket 1 \rrbracket_{A_{\eta}}=1^{\eta}$, 【head $\rrbracket_{A_{\eta}}$ returns the $\eta$ first digits of bit-strings (of size $(n+1) \eta)$ whereas $\llbracket$ tail $_{n} \rrbracket_{A_{\eta}}$ returns the last $n \eta$ digits.

We emphasize that no tags are added to messages. Tags - and in particular tags under encryption - would be harmful to the $\approx_{E_{\text {sym }} \text {-soundness. Indeed we expect }}$ that the formal equivalence $\nu a, b .\{x=\operatorname{enc}(a, b), y=b\} \approx_{E_{\text {sym }}} \nu a, b, c .\{x=$ enc $(a, b), y=c\}$ also holds in the computational world; but this would not be the case if $a$ is tagged before encryption. In case $a$ was tagged before encryption, an adversary could use the tag to check the success of decrypting enc $(a, b)$ with $b$.

For simplicity we assume without loss of generality that encryption keys have the same size $\eta$ as blocks of data. We also assume that keys are generated according to the uniform distribution.

It is not difficult to prove that the above implementation is unconditionally $=E_{\text {sym }}$-sound (by induction on the structure of terms and equational proofs), that is, every true formal equality holds with probability 1 in the concrete world. We note that the equation $\operatorname{enc}_{n}\left(\operatorname{dec}_{n}(x, y), y\right)=x$ is satisfied because encryption by a given key is length-preserving and injective, hence also surjective.

Before studying the $\approx_{E_{\text {sym }}}$-soundness, we need to characterize statically equivalent frames. Specifically, we show that this theory is transparent.

Proposition 9. Let $\varphi$ be a closed frame. There exists a transparent frame $\bar{\varphi}$ such that $\varphi \approx_{E_{\text {sym }}} \bar{\varphi}$.

The proof of Proposition 9 relies on the following Lemma 10, that is used stepwise to rewrite a frame into a transparent frame.

Lemma 10. Let $\varphi$ be a closed frame in $\mathcal{R}$-normal form. Let $T$ be a subterm of $\varphi$ of the form $T=\operatorname{enc}(U, V), T=\operatorname{dec}(U, V), T=\operatorname{head}(V)$ or, $T=\operatorname{tail}(V)$ and $n$ a fresh name of the same sort than $T$. Assume that $V$ is not deducible from $\varphi$, that is, $\varphi \nvdash_{E_{\mathrm{sym}}} V$. Then we have that

$$
\varphi \approx_{E_{\text {sym }}} \varphi^{\prime}
$$

where $\varphi^{\prime}=\varphi\{T \mapsto n\}$ is obtained by replacing every occurrence of $T$ in $\varphi$ with $n$.

The proof of Lemma 10 is given in Appendix C. We prove Proposition 9 by applying this lemma repeatedly on an initial frame $\varphi$. The procedure terminates as each rewriting step decreases the total size of non-deducible subterms in the frame. Besides, the resulting frame $\bar{\varphi}$ is transparent. Indeed, by contradiction, 
suppose that $\bar{\varphi}$ is not transparent; define $T$ as the father of the largest nondeducible subterm of $\varphi$; it is easy to see that $T$ is necessarily of the form $T=$ enc $(U, V), T=\operatorname{dec}(U, V), T=\operatorname{head}(V)$ or $T=\operatorname{tail}(V)$ with $\varphi \forall_{E_{\text {sym }}} V$; thus Lemma 10 applies.

Note that for any subterm $W, \varphi \nvdash_{E_{\text {sym }}} W$ implies $\varphi\{T \mapsto n\} \forall_{E_{\text {sym }}} W\{T \mapsto$ $n\}$. As a consequence, the procedure above yields a unique transparent frame $\bar{\varphi}$ (modulo renaming), no matter in which order the subterms $T$ are substituted.

Provided that $\vdash_{E_{\text {sym }}}$ is decidable ${ }^{1}$, the above procedure for associating transparent frames to frames is effective. Thus, as noticed in Section 4.2, we obtain another proof of the decidability of $\approx_{E_{\text {sym }}}$ using Proposition 4 . Notice that statically equivalent transparent frames may not be equal modulo renaming: consider for instance $\{x=\operatorname{enc}(a, b), y=b\} \approx_{E_{\text {sym }}}\{x=c, y=b\}$.

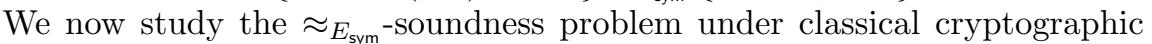
assumptions. Standard assumptions on ciphers include the notions of super pseudo-random permutation (SPRP) and several notions of indistinguishability (IND-P $i$-C $j, i, j=0,1,2)$. In particular, IND-P1-C1 denotes the indistinguishability against lunchtime chosen-plaintext and chosen-ciphertext attacks. These notions and the relations between them have been studied notably in [37].

Initially, the SPRP and IND-P1-C1 assumptions apply to (block) ciphers specialized to plaintexts of a given size. Interestingly, this is not sufficient to imply $\approx_{E_{\text {sym }} \text {-soundness for frames which contain plaintexts of heterogeneous }}$ sizes, encrypted under the same key. Thus we introduce a strengthened version of IND-P1-C1, applying to a collection of ciphers $\left(\mathcal{E}_{\eta, n}, \mathcal{D}_{\eta, n}\right)$, where $\eta$ is the complexity parameter and $n \geq 0$ is the number of blocks of size $\eta$ contained in plaintexts and ciphertexts. One may note that there exist operation modes which turn a fixed size block cipher realizing SPRP into a cipher which handles variable length inputs while preserving SPRP. We refer the reader to [38] for an example of such a mode and further references.

We define the $\omega$-IND-P1-C1 assumption by considering the following experiment $\mathcal{G}_{\eta}$ with a 2 -stage adversary $\mathcal{A}=\left(\mathcal{A}_{1}, \mathcal{A}_{2}\right)$ :

- first a key $k$ is randomly chosen from $\{0,1\}^{\eta}$;

- (Stage 1) $\mathcal{A}_{1}$ is given access to the encryption oracles $\mathcal{E}_{\eta, n}(\cdot, k)$ and the decryption oracles $\mathcal{D}_{\eta, n}(\cdot, k)$; it outputs two plaintexts $m_{0}, m_{1} \in\{0,1\}^{n_{0} \eta}$ for some $n_{0}$, and possibly some data $d$;

- (Stage 2) a random bit $b \in\{0,1\}$ is drawn; $\mathcal{A}_{2}$ receives the data $d$, the challenge ciphertext $c=\mathcal{E}_{\eta, n_{0}}\left(m_{b}, k\right)$ and outputs a bit $b^{\prime}$;

- $\mathcal{A}$ is successful in $\mathcal{G}_{\eta}$ iff $b=b^{\prime}$ and it has never submitted $m_{0}$ or $m_{1}$ to an encryption oracle, nor $c$ to a decryption oracle.

\footnotetext{
${ }^{1}$ A classical characterization of deducibility, entailing its decidability, is detailed in Lemma 23 of Appendix C.
} 
Define the advantage of $\mathcal{A}$ as

$$
\operatorname{Adv}_{\mathcal{A}}^{\omega-\text { IND-P1-C1 }}(\eta)=2 \times \mathbb{P}\left[\mathcal{A} \text { is successful in } \mathcal{G}_{\eta}\right]-1
$$

The $\omega$-IND-P1-C1 assumption holds for $\left(\mathcal{E}_{\eta, n}, \mathcal{D}_{\eta, n}\right)$ iff the advantage of any probabilistic polynomial-time adversary is negligible. It holds for the inverse of the encryption scheme iff it holds for the collection of ciphers $\left(\mathcal{D}_{\eta, n}, \mathcal{E}_{\eta, n}\right)$.

As in previous work $[4,13,18,23]$, we restrict frames to those with only atomic keys and no encryption cycles. Specifically, a closed frame $\varphi$ has only atomic keys if for all subterms enc $n_{n}(u, v)$ and $\operatorname{dec}_{n}(u, v)$ of $\varphi, v$ is a name. Given two (atomic) keys $k_{1}$ and $k_{2}$, we say that $k_{1}$ encrypts $k_{2}$ in $\varphi$, written $k_{1}>_{\varphi} k_{2}$, iff there exists a subterm $U$ of $\varphi$ of the form $U=\operatorname{enc}_{n}\left(T, k_{1}\right)$ or $U=\operatorname{dec}_{n}\left(T, k_{1}\right)$ such that $k_{2}$ appears in $T$ not used as a key, that is, $k_{2}$ appears in $T$ at a position which is not the right-hand argument of a enc $n_{n^{\prime}}$ or a $\operatorname{dec}_{n^{\prime}}$. An encryption cycle is a tuple $k_{1} \ldots k_{m}$ such that $k_{1}>_{\varphi} \ldots>_{\varphi} k_{m}>_{\varphi} k_{1}$.

The effect of the condition "not used as a key" is to allow considering more terms as free of encryption cycles, for instance enc ${ }_{n}\left(\mathrm{enc}_{n}(a, k), k\right)$. This improvement is already suggested in [4].

We now state our $\approx_{E_{\text {sym }} \text {-soundness theorem. A closed frame is well-formed }}$ iff its $\mathcal{R}$-normal form has only atomic keys, contains no encryption cycles and uses no head and tail symbols.

Theorem 11 ( $\approx_{E_{\mathrm{sym}}}$-soundness). Let $\varphi_{1}$ and $\varphi_{2}$ be two well-formed frames of the same domain. Assume that the concrete implementations for the encryption and its inverse satisfy both the $\omega-I N D-P 1-C 1$ assumption. If $\varphi_{1} \approx_{E_{\mathrm{sym}}} \varphi_{2}$ then $\left(\llbracket \varphi_{1} \rrbracket_{A_{\eta}}\right) \approx\left(\llbracket \varphi_{2} \rrbracket_{A_{\eta}}\right)$.

Before proving Theorem 11, we establish a computational counterpart to Lemma 10.

Lemma 12. Let $\varphi$ be a closed frame in $\mathcal{R}$-normal form, with only atomic keys and no encryption cycles. Let $T$ be a subterm of $\varphi$ of the form $T=\operatorname{enc}(U, k)$ (respectively $T=\operatorname{dec}(U, k)$ ), with $k$ name of sort Data, and $n$ a fresh name of the same sort as T. Assume that

- the only occurrences of $k$ in $\varphi$ are in the positions of an encryption or decryption key: enc $(., k)$ or $\operatorname{dec}(., k)$;

- $T$ itself does not appear under an encryption or a decryption with $k$;

- the concrete implementations for the encryption and its inverse satisfy both the $\omega-I N D-P 1-C 1$ assumption.

Then we have that

$$
\left(\llbracket \varphi \rrbracket_{A_{\eta}}\right) \approx\left(\llbracket \varphi^{\prime} \rrbracket_{A_{\eta}}\right)
$$

where $\varphi^{\prime}=\varphi\{T \mapsto n\}$ is obtained by replacing every occurrence of $T$ in $\varphi$ with $n$. 
Notice that the hypothesis of Lemma 12 are stronger than its formal version, Lemma 10. For instance the encryption key $k$ is required to be atomic; the first condition on $k$ implies that $k$ is not deducible from $\varphi$. Also nothing is said about head and tail symbols.

Proof (of Lemma 12). Before proving the lemma, let us consider the example of a well-formed frame $\varphi_{1}=\left\{x_{1}=\operatorname{enc}\left(T_{1}, k\right), x_{2}=\operatorname{enc}\left(T_{2}, k\right)\right\}$, where $k$ does not appear in $T_{1}, T_{2}$, and $T_{1} \neq_{E_{\text {sym }}} T_{2}$. This frame is statically equivalent to $\varphi_{2}=\left\{x_{1}=n_{1} ; x_{2}=n_{2}\right\}$. Our problem here is to prove that $\llbracket \varphi_{1} \rrbracket$ and $\llbracket \varphi_{2} \rrbracket$ are actually indistinguishable. It is not hard to see that this will be the case if and only if the probability that $T_{1}$ and $T_{2}$ have the same concrete value is negligible. A consequence of this phenomenon is intuitively that we need to prove Lemma 12 and - at least - a limited form of $={ }_{E_{\mathrm{sym}}}$-faithfulness at the same time.

Formally, let us write $|\varphi|_{e}$ and $|T|_{e}$ for the number of distinct subterms with head symbols enc or dec, occurring respectively in a frame $\varphi$ and a term $T$. Let $P_{n}$ and $Q_{n}$ be the two properties:

$\left(P_{n}\right)$ Lemma 12 holds provided that $|\varphi|_{e} \leq n$ :

For every $\mathcal{R}$-normal, closed frame $\varphi$ containing only atomic keys, no encryption cycles, and such that $|\varphi|_{e} \leq n$, for every maximal subterm $T$ of $\varphi$ of the form $T=\operatorname{enc}(U, k)$ or $T=\operatorname{dec}(U, k)$, for every fresh name $n$ of the appriopriate sort, if the only occurrences of $k$ in $\varphi$ are in key positions (i.e. enc $(., k)$ or $\operatorname{dec}(., k))$, then $\left(\llbracket \varphi \rrbracket_{A_{\eta}}\right) \approx$ $\left(\llbracket \varphi\{T \mapsto n\} \rrbracket_{A_{\eta}}\right)$.

$\left(Q_{n}\right)$ For all $\mathcal{R}$-normal terms $T_{1}, T_{2}$ of the same sort such that: $T_{1}, T_{2}$ have only atomic keys, the frame $\varphi=\left\{x=T_{1}, y=T_{2}\right\}$ has no encryption cycles, $T_{1} \neq T_{2}$ and $|\varphi|_{e} \leq n$, the probability $\mathbb{P}\left[e_{1}, e_{2} \leftarrow \llbracket T_{1}, T_{2} \rrbracket_{A_{\eta}} ; e_{1}=e_{2}\right]$ is negligible.

We prove $P_{n}$ and $Q_{n}$ by mutual induction on $n$, that is, more precisely we prove the four statements: (S1) $P_{0}$, (S2) $P_{n+1} \Leftarrow Q_{n}$, (S3) $Q_{0}$, (S4) $Q_{n+1} \Leftarrow$ $\left(P_{n+1}\right.$ and $\left.Q_{n}\right)$.

(S1) $P_{0}$ is vacuously true.

(S2) $P_{n+1} \Leftarrow Q_{n}$. Let $T^{0}=$ enc $_{n_{0}}(U, k)$ be a subterm of $\varphi, k$ and $n$ two names all satisfying the conditions of Lemma 12. (Naturally, the case of $T^{0}=$ $\operatorname{dec}_{n_{0}}(U, k)$ is similar.) Let $\varphi=\left\{x_{1}=T_{1}^{0}, \ldots, x_{n}=T_{n}^{0}\right\}$.

Provided an adversary $\mathcal{A}$ able to distinguish $\left(\llbracket \varphi \rrbracket_{A_{\eta}}\right)$ and $\left(\llbracket \varphi^{\prime} \rrbracket_{A_{\eta}}\right)$, we build an adversary $\mathcal{B}$ against the $\omega$-IND-P1-C1 assumption on encryption, described as follows:

1. for each name $a$ of sort $s$ appearing in $\varphi$, draw a value $\widehat{a} \stackrel{R}{\longleftarrow} \llbracket s \rrbracket_{A_{\eta}}$;

2. draw a value $\widehat{a_{0}} \stackrel{R}{\longleftarrow} \llbracket s \rrbracket_{A_{\eta}}$ for some fresh name $a_{0}$ of sort List $_{n_{0}}$;

3. for each $x_{i}(1 \leq i \leq n)$ of sort $s_{i}$, compute $\widehat{T_{i}^{0}} \in \llbracket s_{i} \rrbracket_{A}$ recursively as 
follows:

$$
\begin{aligned}
& \widehat{\mathrm{enc}_{n}(T, k)}=\mathcal{E}_{n}(\widehat{T}) \text { if } T \neq U \\
& \widehat{\text { enc }_{n_{0}}(U, k)}=\mathcal{E}^{*}\left(\widehat{U}, \widehat{a_{0}}\right) \\
& \widehat{\operatorname{dec}_{n}(T, k)}=\mathcal{D}_{n}(\widehat{T}) \\
& f\left(\widehat{T_{1}, \ldots,} T_{n}\right)=\llbracket f \rrbracket_{A_{\eta}}\left(\widehat{T_{1}}, \ldots, \widehat{T_{n}}\right) \quad \text { in the remaining cases }
\end{aligned}
$$

where we have written $\mathcal{E}_{n}($.$) and \mathcal{D}_{n}($.$) for the encryption and decryp-$ tion oracles of the $\omega$-IND-P1-C1 game, and $\mathcal{E}^{*}\left(\widehat{U}, \widehat{a_{0}}\right)$ for the challenge ciphertext, obtained after submitting the two plaintexts $\widehat{U}$ and $\widehat{a_{0}}$. Since $T^{0}=$ enc $_{n_{0}}(U, k)$ is not a subterm of an encryption or a decryption with $k$, we may assume that $\mathcal{E}^{*}\left(\widehat{U}, \widehat{a_{0}}\right)$ is computed only once, after every call to $\mathcal{E}_{n}($.$) and \mathcal{D}_{n}($.$) ;$

4. submit the concrete frame $\left\{x_{1}=\widehat{T_{1}}, \ldots, x_{n}=\widehat{T_{n}}\right\}$ to $\mathcal{A}$ and return the same answer.

The distribution computed by $\mathcal{B}$ and submitted to $\mathcal{A}$ equals either $\left(\llbracket \varphi \rrbracket_{A_{\eta}}\right)$ or $\left(\llbracket \varphi^{\prime} \rrbracket_{A_{\eta}}\right)$ depending on whichever $\mathcal{E}^{*}\left(\widehat{U}, \widehat{a_{0}}\right)$ is the encryption of $\widehat{U}$, or respectively, that of $\widehat{a_{0}}$ (in the latter case $\mathcal{E}^{*}\left(\widehat{U}, \widehat{a_{0}}\right)=\mathcal{E}_{n_{0}}\left(\widehat{a_{0}}\right)$ is simply a random number). Thus the probability that $\mathcal{B}$ guesses the right answer is the same as $\mathcal{A}$. Now it may happen that $\mathcal{B}$ does not meet the second requirement for winning the $\omega$-IND-P1-C1 game, that is: (i) there exists a subterm enc $n_{n_{0}}(T, k)$ such that $T \neq U$ and $\widehat{T} \in\left\{\widehat{U}, \widehat{a_{0}}\right\}$ or (ii) there exists a subterm $\operatorname{dec}_{n_{0}}(T, k)$ such that $\widehat{T}=\mathcal{E}^{*}\left(\widehat{U}, \widehat{a_{0}}\right)$.

For (i), the probability that $\widehat{T}=\widehat{a_{0}}$ is negligible by construction. Moreover, as $T$ and $T^{0}=\operatorname{enc}_{n_{0}}(U, k)$ are two subterms of $\varphi$ and $T^{0}$ is not a subterm of $T$, the frame $\varphi^{\prime}=\{x=T, y=U\}$ has no encryption cycles and $\left|\varphi^{\prime}\right|_{e}<|\varphi|_{e}=n+1$. The induction hypothesis $Q_{n}$ implies that the probability for $\widehat{T}=\widehat{U}$ is negligible.

As for (ii), if the challenge ciphertext $\mathcal{E}^{*}\left(\widehat{U}, \widehat{a_{0}}\right)$ is the encryption of its second argument, that is $\mathcal{E}_{n_{0}}\left(\widehat{a_{0}}\right)$, then the probability for $\widehat{T}=\mathcal{E}^{*}\left(\widehat{U}, \widehat{a_{0}}\right)$ is negligible; otherwise $\mathcal{E}^{*}\left(\widehat{U}, \widehat{a_{0}}\right)=\mathcal{E}_{n_{0}}(\widehat{U})$. Recall that $T^{0}=$ enc $_{n_{0}}(U, k)$ is in $\mathcal{R}$-normal form, thus $U \neq \operatorname{dec}_{n_{0}}(T, k)$. As $T^{0}$ and $\operatorname{dec}_{n_{0}}(T, k)$ are two subterms of $\varphi$ and $T^{0}$ is not a subterm of $\operatorname{dec}_{n_{0}}(T, k)$, the frame $\varphi^{\prime}=\left\{x=U, y=\operatorname{dec}_{n_{0}}(T, k)\right\}$ has no encryption cycles and $\left|\varphi^{\prime}\right|_{e}<|\varphi|_{e}=n+1$, hence the induction hypothesis $Q_{n}$ implies that the probability for $\widehat{T}=\mathcal{E}_{n_{0}}(\widehat{U})$ is negligible.

To simplify the case analysis of (S3) and (S4), it is convenient to introduce the following lemma:

Lemma 13. Let $T_{1}, T_{2}$ be two terms of sort List ${ }_{j}$. Define for each $1 \leq i \leq j$, the $i$-th projection of a term $T$ of sort List ${ }_{j}$, by:

$$
\left.\pi_{i}(T)=\operatorname{head}(\underbrace{\text { tail }(\ldots \text { tail }}_{i-1 \text { times }}(T))\right)
$$


Then (i) $T_{1}={ }_{E_{\mathrm{sym}}} T_{2}$ iff for all $1 \leq i \leq j, \pi_{i}\left(T_{1}\right)={ }_{E_{\mathrm{sym}}} \pi_{i}\left(T_{2}\right)$ and moreover (ii) $\mathbb{P}\left[e 1, e 2 \leftarrow \llbracket T_{1}, T_{2} \rrbracket_{A_{\eta}} ; e_{1}=e_{2}\right]$ is negligible iff for all $1 \leq i \leq j$,

$$
\mathbb{P}\left[e_{1}^{i}, e_{2}^{i} \leftarrow \llbracket \pi_{i}\left(T_{1}\right) \downarrow_{\mathcal{R}}, \pi_{i}\left(T_{2}\right) \downarrow_{\mathcal{R}} \rrbracket_{A_{\eta}} ; e_{1}^{i}=e_{2}^{i}\right]
$$

is negligible.

(The notation $T \downarrow_{\mathcal{R}}$ stands for the $\mathcal{R}$-normal form of $T$.)

Thanks to this lemma, it is sufficient to prove (S3) and (S4) for $T_{1}$ and $T_{2}$ of sort Data and in $\mathcal{R}$-normal form. (Indeed notice that if $\varphi=\left\{x=T_{1}, y=T_{2}\right\}$ has no encryption cycles, then $\varphi^{\prime}=\left\{x^{\prime}=\pi_{i}\left(T_{1}\right) \downarrow_{\mathcal{R}}, y^{\prime}=\pi_{i}\left(T_{2}\right) \downarrow_{\mathcal{R}}\right\}$ has no encryption cycles and $\left|\varphi^{\prime}\right|_{e} \leq|\varphi|_{e}$.)

Given the sorting system and the rewriting rules, a $\mathcal{R}$-reduced term $T$ of sort Data may only be of the following forms:

1. a constant: 0 or 1 ,

2. a name of sort Data: $T=a$,

3. a projection of name of sort List $_{j}: T=\pi_{i}(a)(1 \leq i \leq j)$,

4. a projection of a encryption/decryption of sort List $_{j}: T=\pi_{i}(\operatorname{enc}(U, V))$ with $U \notin\left\{\operatorname{dec}\left(T^{\prime}, V\right)\right\}$ or $T=\pi_{i}(\operatorname{dec}(U, V))$ with $U \notin\left\{\operatorname{enc}\left(T^{\prime}, V\right)\right\}$.

(S3) $Q_{0}$. As $T_{1}$ and $T_{2}$ contain no encryption/decryption symbol, only the cases $1-3$ of the case analysis above can occur; the property follows directly.

(S4) $Q_{n+1} \Leftarrow\left(P_{n+1}\right.$ and $\left.Q_{n}\right)$. Let $T_{1}$ and $T_{2}$ be two distinct closed normal terms and $\varphi=\left\{x=T_{1}, y=T_{2}\right\}$. Assume that $\varphi$ has no encryption cycles nor composed keys, and $|\varphi|_{e}=n+1$.

1. If one of the two terms - say $T_{1}$ - is of the form 1 (constant), 2 (name) or 3 (projection of a name). Then $T_{2}$ is of the form 4 , for instance $T_{2}=$ $\pi_{i}(\operatorname{enc}(U, k))$ with $U \notin\left\{\operatorname{dec}\left(T^{\prime}, k\right)\right\}$.

(a) If $T_{1} \neq k$, by $P_{n+1}$, we have $\left(\llbracket \varphi \rrbracket A_{\eta}\right) \approx\left(\llbracket\left\{x=T_{1}, y=\pi_{i}(a)\right\} \rrbracket_{A_{\eta}}\right)$ for some fresh name $a$. In particular, the probability for the two components $x$ and $y$ to be equal is negligible.

(b) If $T_{1}=k$, assume that $T_{1}$ and $T_{2}$ yields the same concrete value with significant probability. Let List $_{n_{0}}$ be the sort of $U$. We build an adversary $\mathcal{A}$ to the $\omega$-IND-P1-C1 game as follows:

i. for each name $a$ of sort $s$ appearing in $T_{2}$, draw a value $\widehat{a} \stackrel{R}{\longleftarrow}$ $\llbracket s \rrbracket_{A_{\eta}} ;$

ii. draw a value $\widehat{a_{0}} \stackrel{R}{\longleftarrow} \llbracket s \rrbracket_{A_{\eta}}$ for some fresh name $a_{0}$ of sort List $_{n_{0}}$;

iii. compute $\widehat{T_{2}}$ recursively as follows:

$$
\begin{aligned}
\widehat{\operatorname{enc}(T, k)} & =\mathcal{E}_{n}(\widehat{T}) \text { if } T \neq U \\
\underset{\operatorname{enc}_{n_{0}}(U, k)}{ } & =\mathcal{E}^{*}\left(\widehat{U}, \widehat{a_{0}}\right) \\
\operatorname{dec}_{n}(T, k) & =\mathcal{D}_{n}(\widehat{T}) \\
f\left(\widehat{V_{1}, \ldots,}, V_{n}\right) & =\llbracket f \rrbracket_{A_{\eta}}\left(\widehat{V_{1}}, \ldots, \widehat{V_{n}}\right) \quad \text { in the remaining cases }
\end{aligned}
$$


using the same conventions as before;

iv. if $\left.\mathcal{E}_{n_{0}}\left(\widehat{U}, \widehat{T_{2}}\right)=\mathcal{E}^{*}\left(\widehat{U}, \widehat{a_{0}}\right)\right)$, return 0 , otherwise return 1 .

$\mathcal{A}$ guesses the correct answer with non-negligible probability. As before, we use the property $Q_{n}$ to conclude that its advantage is non-negligible.

2. Suppose $T_{1}=\pi_{i_{1}}\left(\operatorname{enc}\left(u_{1}, k_{1}\right)\right)$ and $T_{2}=\pi_{i_{2}}\left(\operatorname{enc}\left(u_{2}, k_{2}\right)\right)$ (the 3 other cases with decryption symbols are similar). As $\varphi$ has no encryption cycle, we may assume for instance that $k_{1}$ is maximal for $<_{\varphi}$. Let $T$ be a maximal subterm of the form enc $\left(U, k_{1}\right)$ or $\operatorname{dec}\left(U, k_{1}\right)$ in $\varphi$. By $P_{n+1}$, we have $\left(\llbracket \varphi \rrbracket_{A_{\eta}}\right) \approx\left(\llbracket \varphi^{\prime} \rrbracket_{A_{\eta}}\right)$ where $\varphi^{\prime}=\varphi\{T \mapsto a\}=\left\{x=T_{1}^{\prime}, y=T_{2}^{\prime}\right\}$ for some fresh name $a$. We then apply $Q_{n}$ to $T_{1}^{\prime}$ and $T_{2}^{\prime}$.

Proof (of Lemma 13). Point (i) is easily shown by induction on $i$, using the equations of $E_{\text {sym }}$. For (ii), notice that:

$$
\mathbb{P}\left[e 1, e 2 \leftarrow \llbracket T_{1}, T_{2} \rrbracket_{A_{\eta}} ; e_{1}=e_{2}\right] \leq \sum_{i=1}^{j} \mathbb{P}\left[e_{1}^{i}, e_{2}^{i} \leftarrow \llbracket \pi_{i}\left(T_{1}\right), \pi_{i}\left(T_{2}\right) \rrbracket_{A_{\eta}} ; e_{1}^{i}=e_{2}^{i}\right]
$$

and

$$
\forall i, \quad \mathbb{P}\left[e 1, e 2 \leftarrow \llbracket T_{1}, T_{2} \rrbracket_{A_{\eta}} ; e_{1}=e_{2}\right] \geq \mathbb{P}\left[e_{1}^{i}, e_{2}^{i} \leftarrow \llbracket \pi_{i}\left(T_{1}\right), \pi_{i}\left(T_{2}\right) \rrbracket_{A_{\eta}} ; e_{1}^{i}=e_{2}^{i}\right]
$$

Besides it is clear from the unconditional $=_{E_{\text {sym }}}$-soundness, that for any $T_{1}, T_{2}$ :

$$
\mathbb{P}\left[e 1, e 2 \leftarrow \llbracket T_{1}, T_{2} \rrbracket_{A_{\eta}} ; e_{1}=e_{2}\right]=\mathbb{P}\left[e 1, e 2 \leftarrow \llbracket T_{1} \downarrow_{\mathcal{R}}, T_{2} \downarrow_{\mathcal{R}} \rrbracket_{A_{\eta}} ; e_{1}=e_{2}\right]
$$

Proof (of Theorem 11). Thanks to the (unconditional) $=_{E_{\text {sym }}}$-soundness, it is enough to prove the property on frames in $\mathcal{R}$-normal form.

We begin by proving the following lemma:

Lemma 14. Assume that the concrete implementations for the encryption and its inverse satisfy both the $\omega-I N D-P 1-C 1$ assumption. For every well-formed $\mathcal{R}$ normal frame $\varphi,\left(\llbracket \varphi \rrbracket_{A_{\eta}}\right) \approx\left(\llbracket \bar{\varphi} \rrbracket_{A_{\eta}}\right)$ where $\bar{\varphi}$ is the transparent frame associated to $\varphi$ following the algorithmic proof of Proposition 9 (this transparent frame is uniquely defined modulo renaming of names.).

Now recall that by Proposition 5 and since $\varphi \approx \bar{\varphi}$, we have:

$$
\llbracket \bar{\varphi} \rrbracket A_{A_{\eta}}=\llbracket \bar{\varphi} \rrbracket_{A_{\eta}}^{\text {ideal }}=\llbracket \varphi \rrbracket_{A_{\eta}}^{\text {ideal }}
$$

Therefore the soundness criterion holds for well-formed $\mathcal{R}$-normal frames and we conclude by Proposition 3.

Notice that the use of the ideal semantics could not be easily avoided as two statically equivalent transparent frames may not be equal modulo renaming of bound names. 
Proof (of Lemma 14). We prove the property by induction on the number $m$ of encryptions and decryptions by non-deducible keys in $\varphi$.

If $m=0$, by the well-formedness condition, $\varphi$ is already a transparent frame.

Suppose that $m>0$. As $\varphi$ has no encryption cycle, we choose a nondeducible (atomic) key $k$ appearing in $\varphi$, such that $k$ is maximal for the encryption relation $>_{\varphi}$.

As $k$ is not deducible, is maximal for $>_{\varphi}$ and $\varphi$ contains no head and tail symbols, the only occurrences of $k$ in $\varphi$ are as encryption or decryption keys. Let $T$ be a maximal subterm of $\varphi$ of the form $T=\operatorname{enc}(U, k)$ or $T=\operatorname{dec}(U, k)$. We apply Lemma 12 on $\varphi$ and $T$ and conclude by induction hypothesis on the obtained frame $\varphi^{\prime}$.

Note on the cryptographic assumptions.. Cryptographic assumptions of Theorem 11 may appear strong compared to existing work on passive adversaries [4, 13]. This seems unavoidable when we allow frames to contain both encryption and decryption symbols.

In the case where the two frames to be compared contain no decryption symbols, our proofs are easily adapted to work when the encryption scheme is $\omega$-IND-P1-C0 only, where $\omega$-IND-P1-C0 is defined similarly to $\omega$-IND-P1C1 except that the adversary has no access to the decryption oracle. Such an assumption is realizable in practice using a variable-input-length cipher [39, 38].

Finally, it should be possible to recover the classical assumption IND-P1C1 by modeling the ECB mode (Electronic Code Book). Consider two new symbols enc : Data $\times$ Data $\rightarrow$ Data and dec : Data $\times$ Data $\rightarrow$ Data, and define the symbols enc ${ }_{n}$ and $\operatorname{dec}_{n}$ (formally and concretely) recursively by

$$
\begin{aligned}
& \operatorname{enc}_{n+1}(x, y)=\operatorname{cons}_{n}\left(\operatorname{enc}\left(\operatorname{head}_{n}(x), y\right), \operatorname{enc}_{n}\left(\operatorname{tail}_{n}(x), y\right)\right) \text { and } \\
& \operatorname{dec}_{n+1}(x, y)=\operatorname{cons}_{n}\left(\operatorname{dec}\left(\operatorname{head}_{n}(x), y\right), \operatorname{dec}_{n}\left(\operatorname{tail}_{n}(x), y\right)\right)
\end{aligned}
$$

together with the equations

$$
\begin{aligned}
& \operatorname{dec}(\operatorname{enc}(x, y), y)=x \\
& \operatorname{enc}(\operatorname{dec}(x, y), y)=y
\end{aligned}
$$

Define well-formed frames as those of which the normal forms contain no encryption cycles. Then, similar techniques can be applied to show that $\approx_{E_{\text {sym }}}$ soundness holds for well-formed frames as soon as the implementations for enc and dec are both IND-P1-C1, or equivalently [37], enc is SPRP.

Note on the well-formedness assumptions.. We may also note that it is possible to slightly relax the assumptions of well-formedness of frames. In particular we could allow encryption cycles on deducible keys and for instance allow the frame $\left\{x=\operatorname{enc}\left(k_{1}, k_{2}\right), y=\operatorname{enc}\left(k_{2}, k_{1}\right), z=k_{1}\right\}$ which is currently discarded. As these extensions are not essential for our results we prefer to avoid unnecessary clutter and keep the definitions simple. 


\section{Conclusion and future work}

In this paper we developed a general framework for relating formal and computational models of security protocols in the presence of a passive attacker. These are the first results on abstract models allowing arbitrary equational theories. We define the soundness and faithfulness of cryptographic implementations with respect to abstract models. We also provide a soundness criterion which is not only sufficient but also necessary for many theories. Finally, we provide new soundness results for the exclusive OR and a theory of ciphers and lists.

A direction for further work is to study the soundness of other theories. An interesting case is the combination of the two theories considered in this paper, that is modeling the exclusive OR, ciphers and lists. Another interesting open problem is to generalize the notion of transparent frames so as to include probabilistic encryption, while retaining the essential properties of transparent frames. Finally, an ambitious extension is to consider the case of an active attacker in presence of general equational theories.

Acknowledgments.. We would like to thank the anonymous reviewers for their helpful suggestions. This work was partially supported by the ACI JC 9005, the ARA SSIA FormaCrypt and the ANR SESUR AVOTÉ.

\section{References}

[1] M. Baudet, V. Cortier, S. Kremer, Computationally sound implementations of equational theories against passive adversaries, in: Proc. 32nd International Colloquium on Automata, Languages and Programming (ICALP'05), Vol. 3580 of LNCS, Springer, 2005, pp. 652-663.

[2] D. Dolev, A. C. Yao, On the security of public key protocols, IEEE Transactions on Information Theory IT-29 (12) (1983) 198-208.

[3] S. Goldwasser, S. Micali, Probabilistic encryption, Journal of Computer and System Sciences 28 (1984) 270-299.

[4] M. Abadi, P. Rogaway, Reconciling two views of cryptography (the computational soundness of formal encryption), in: Proc. 1st IFIP International Conference on Theoretical Computer Science (IFIP-TCS'00), Vol. 1872 of LNCS, 2000, pp. 3-22.

[5] H. Comon, V. Shmatikov, Is it possible to decide whether a cryptographic protocol is secure or not?, Journal of Telecommunications and Information Technology (4/2002) 5-15.

[6] V. Cortier, S. Delaune, P. Lafourcade, A survey of algebraic properties used in cryptographic protocols, Journal of Computer Security 14 (1) (2006) 143. 
[7] M. Abadi, C. Fournet, Mobile values, new names, and secure communications, in: Proc. 28th Annual ACM Symposium on Principles of Programming Languages (POPL'01), 2001, pp. 104-115.

[8] R. Corin, J. Doumen, S. Etalle, Analysing password protocol security against off-line dictionary attacks, in: Proc. 2nd International Workshop on Security Issues with Petri Nets and other Computational Models (WISP'04), Vol. 121 of ENTCS, 2005, pp. 47-63.

[9] M. Baudet, Deciding security of protocols against off-line guessing attacks, in: Proc. 12th ACM Conference on Computer and Communications Security (CCS'05), ACM Press, 2005, pp. 16-25.

[10] M. Abadi, V. Cortier, Deciding knowledge in security protocols under equational theories, in: Proc. 31st International Colloquium on Automata, Languages and Programming (ICALP'04), Vol. 3142 of LNCS, 2004, pp. 46-58.

[11] B. Blanchet, Automatic proof of strong secrecy for security protocols, in: Proc. 25th IEEE Symposium on Security and Privacy (SSP'04), 2004, pp. 86-100.

[12] P. Adão, G. Bana, A. Scedrov, Computational and information-theoretic soundness and completeness of formal encryption, in: Proc. 18th IEEE Computer Security Foundations Workshop (CSFW'05), 2005, pp. 170-184.

[13] D. Micciancio, B. Warinschi, Completeness theorems for the AbadiRogaway logic of encrypted expressions, Journal of Computer Security 12 (1) (2004) 99-129.

[14] P. Laud, Computationally secure information flow, Ph.D. thesis, Universität des Saarlandes (2002).

[15] P. Laud, R. Corin, Sound computational interpretation of formal encryption with composed keys, in: Proc. 6th International Conference on Information Security and Cryptology (ICISC'03), Vol. 2971 of LNCS, 2004, pp. $55-66$.

[16] P. Adão, J. Herzog, G. Bana, A. Scedrov, Soundness of formal encryption in the presence of key-cycles, in: Proc. 10th European Symposium on Research in Computer Security (ESORICS'05), Vol. 3679 of LNCS, 2005, pp. 374-396.

[17] M. Backes, B. Pfitzmann, M. Waidner, A composable cryptographic library with nested operations, in: Proc. 10th ACM Conference on Computer and Communications Security (CCS'03), ACM Press, 2003, pp. 220-230.

[18] M. Backes, B. Pfitzmann, Symmetric encryption in a simulatable DolevYao style cryptographic library, in: Proc. 17th IEEE Computer Science Foundations Workshop (CSFW'04), 2004, pp. 204-218. 
[19] M. Backes, B. Pfitzmann, M. Waidner, Symmetric authentication within simulatable cryptographic library, in: Proc. 8th European Symposium on Research in Computer Security (ESORICS'03), LNCS, 2003, pp. 271-290.

[20] V. Cortier, B. Warinschi, Computationally sound, automated proofs for security protocols, in: Proc. 14th European Symposium on Programming (ESOP'05), Vol. 3444 of LNCS, 2005, pp. 157-171.

[21] R. Janvier, Y. Lakhnech, L. Mazaré, Completing the picture: Soundness of formal encryption in the presence of active adversaries, in: Proc. 14th European Symposium on Programming (ESOP'05), Vol. 3444 of LNCS, 2005, pp. 172-185.

[22] R. Canetti, J. Herzog, Universally composable symbolic analysis of mutual authentication and key-exchange protocols (extended abstract), in: Proc. 3rd Theory of Cryptography Conference (TCC'06), Vol. 3876 of LNCS, 2006, pp. 380-403.

[23] P. Laud, Symmetric encryption in automatic analyses for confidentiality against active adversaries, in: Proc. IEEE Symposium on Security and Privacy (SSP'04), 2004, pp. 71-85.

[24] A. Datta, A. Derek, J. C. Mitchell, V. Shmatikov, M. Turuani, Probabilistic Polynomial-time Semantics for a Protocol Security Logic, in: Proc. 32nd International Colloquium on Automata, Languages and Programming, ICALP, Vol. 3580 of LNCS, Springer, 2005, pp. 16-29, lisboa, Portugal.

[25] B. Blanchet, A computationally sound mechanized prover for security protocols, in: IEEE Symposium on Security and Privacy, IEEE Computer Society Press, 2006, pp. 140-154.

[26] M. Backes, B. Pfitzmann, Limits of the cryptographic realization of dolevyao-style xor, in: Proc. 10th European Symposium on Research in Computer Security (ESORICS'05), Vol. 3679 of LNCS, 2005, pp. 336-354.

[27] M. Abadi, B. Warinschi, Password-based encryption analyzed, in: Proc. 32nd International Colloquium on Automata, Languages and Programming (ICALP'05), Vol. 3580 of LNCS, 2005, pp. 664-676.

[28] M. Abadi, M. Baudet, B. Warinschi, Guessing attacks and the computational soundness of static equivalence, in: Proc. 9th International Conference on Foundations of Software Science and Computation Structures (FoSSaCS'06), Vol. 3921 of LNCS, 2006, pp. 398-412.

[29] G. Bana, P. Mohassel, T. Stegers, The computational soundness of formal indistinguishability and static equivalence, in: Proc. 11th Asian Computing Science Conference (ASIAN'06), Vol. 4435 of LNCS, Springer, 2006, pp. 182-196. 
[30] S. Kremer, L. Mazaré, Adaptive soundness of static equivalence, in: Proc. 12th European Symposium on Research in Computer Security (ESORICS'07), Vol. 4734 of LNCS, Springer, 2007, pp. 610-625.

[31] H. Comon-Lundh, V. Cortier, Computational soundness of observational equivalence, in: Proc. 15th ACM Conference on Computer and Communications Security (CCS'08), ACM Press, 2008, pp. 109-118.

[32] M. Abadi, C. Fournet, Mobile values, new names, and secure communication, in: Proc. of the 28th ACM Symposium on Principles of Programming Languages (POPL'01), 2001, pp. 104-115.

[33] S. Hohenberger, The cryptographic impact of groups with infeasible inversion, Master's thesis, MIT (2003).

[34] R. L. Rivest, On the notion of pseudo-free groups, in: Proc. 1st Theory of Cryptography Conference (TCC'04), Vol. 2951 of LNCS, 2004, pp. 505-521.

[35] S. Goldwasser, M. Bellare, Lecture notes on cryptography (2008).

[36] D. Micciancio, The RSA group is pseudo-free, in: Advances in Cryptology - Proc. EUROCRYPT '05, Vol. 3494 of LNCS, 2005, pp. 387-403.

[37] D. H. Phan, D. Pointcheval, About the security of ciphers (semantic security and pseudo-random permutations), in: Proc. Selected Areas in Cryptography (SAC'04), Vol. 3357 of LNCS, 2004, pp. 185-200.

[38] S. Halevi, Invertible universal hashing and the TET encryption mode, in: Advances in Cryptology - Proc. CRYPTO '2007, Vol. 4622 of LNCS, 2007, pp. $412-429$.

[39] M. Bellare, P. Rogaway, On the construction of variable-input-length ciphers, in: Proc. 6th Workshop on Fast Software Encryption (FSE'99), Vol. 1636 of LNCS, 1999, pp. 231-244.

[40] E. Contejean, C. Marché, B. Monate, X. Urbain, The CiME Rewrite Tool, http://cime.lri.fr (2000).

\section{A. General results on static equivalence}

We prove here some general properties of static equivalence concerning free symbols. We first establish a useful interpolation lemma.

Given a term $U=f\left(U_{1}, \ldots, U_{n}\right)$ where $f$ is a free symbol (see Section 2.1) and a name $a$ of the same sort as $U$, the cutting function cut $_{U, a}$ is defined recursively as follows: $\operatorname{cut}_{U, a}(u)=u$ if $u$ is a variable or a name, and

$$
\operatorname{cut}_{U, a}\left(g\left(T_{1}, \ldots, T_{k}\right)\right)=\left\{\begin{array}{l}
a \text { if } g=f, k=n \text { and } \forall 1 \leq i \leq n, U_{i}={ }_{E} T_{i} \\
g\left(\operatorname{cut}_{U, a}\left(T_{1}\right), \ldots, \operatorname{cut}_{U, a}\left(T_{k}\right)\right) \text { otherwise }
\end{array}\right.
$$

Thus, the effect of function $\operatorname{cut}_{U, a}(T)$ is to substitute some (but not all) subterms of $T$ equal to $U$ modulo $E$ with $a$. 
Lemma 15. Let $U=f\left(U_{1}, \ldots, U_{n}\right)$ be a term such that $f$ is a free symbol. Let $a$ be a name of the same sort as $U$. For any two terms $M$ and $N$,

$$
M={ }_{E} N \quad \text { implies } \operatorname{cut}_{U, a}(M)={ }_{E} \operatorname{cut}_{U, a}(N) .
$$

Proof. By Birkhoff's theorem, $M={ }_{E} N$ means that there exist $n \geq 0$ and $M_{0}, \ldots, M_{n}$ such that $M=M_{0} \leftrightarrow_{E} M_{1} \leftrightarrow_{E} M_{n}=N$ where $\leftrightarrow_{E}$ denotes one step of rewriting along one equation in (the generating set of) $E$, oriented in either direction.

To prove the property by induction on $n$, it suffices to consider the case $n=1$. More precisely, assume that there exists an equation $l=r$ in $E$, a position $p$ and a substitution $\theta$ such that $\left.M\right|_{p}=l \theta$ and $N=M[r \theta]_{p}$. By definition of free symbols, we may assume that $f$ does not occur in $l$ and $r$. We consider two cases depending on whether the cutting function cut $_{U, a}$ cuts a subterm above $p$ or not.

- Either there exists a proper prefix $p^{\prime}$ of $p$ such that $\left.M\right|_{p^{\prime}}=f\left(T_{1}, \ldots, T_{k}\right)$ and for all $i, U_{i}=_{E} T_{i}$. We consider the smallest $p^{\prime}$ that satisfies this property. Thus $p=p^{\prime} \cdot i \cdot p^{\prime \prime}$ and $N=M\left[f\left(T_{1}, \ldots, T_{i}[r \theta]_{p^{\prime \prime}}, \ldots, T_{n}\right)_{p^{\prime}}\right]$. Both terms $f\left(T_{1}, \ldots, T_{k}\right)$ and $f\left(T_{1}, \ldots, T_{i}[r \theta]_{p^{\prime \prime}}, \ldots, T_{n}\right)$ are substituted with $a$, thus $\operatorname{cut}_{U, a}(M)=\operatorname{cut}_{U, a}(N)$.

- Or no such cutting position $p^{\prime}$ is a proper prefix of $p$. This means that $\operatorname{cut}_{U, a}\left(M[x]_{p}\right)=\operatorname{cut}_{U, a}\left(N[x]_{p}\right)$ and $\operatorname{cut}_{U, a}(M)=\operatorname{cut}_{U, a}\left(M[x]_{p}\right)\left[\operatorname{cut}_{U, a}(l \theta)\right]_{p}$, where $x$ is a fresh variable. Moreover, $\operatorname{cut}_{U, a}(l \theta)=\operatorname{ccut}_{U, a}(\theta)$ and $\operatorname{cut}_{U, a}(r \theta)=$ $r$ cut $_{U, a}(\theta)$ since $f$ is free. We deduce

$$
\begin{aligned}
& \operatorname{cut}_{U, a}(M)=\operatorname{cut}_{U, a}\left(M[x]_{p}\right)\left[\operatorname{cut}_{U, a}(l \theta)\right]_{p} \\
&= \operatorname{cut}_{U, a}\left(N[x]_{p}\right)[\operatorname{lcut} U, a \\
&=\operatorname{cut}_{U, a}\left(N[x]_{p}\right)\left[\operatorname{rcut}_{U, a}(\theta)\right]_{p} \\
&=\operatorname{cut}_{U, a}(N)
\end{aligned}
$$

Using this lemma, we establish two simple properties of free symbols.

Corollary 16. Let $f$ be a free symbol and $f\left(T_{1}, \ldots, T_{n}\right)$ a term of a nondegenerated type $\tau$.

1. For every $U_{1}, \ldots, U_{n}$ of the appropriate sort,

$$
f\left(T_{1}, \ldots T_{n}\right)={ }_{E} f\left(U_{1}, \ldots, U_{n}\right) \quad \text { iff } \quad \forall i, T_{i}={ }_{E} U_{i} .
$$

2. Let $U$ be a term of sort $\tau$ such that $f$ does not appear in $U$. Then

$$
f\left(T_{1}, \ldots, T_{n}\right) \neq{ }_{E} U \text {. }
$$

Proof. 
1. The right-to-left implication is trivial. Let $T=f\left(T_{1}, \ldots T_{n}\right)$ and $U=$ $f\left(U_{1}, \ldots, U_{n}\right)$. By contradiction, assume that there exists an $i$ such that $T_{i} \neq_{E} U_{i}$. Let $a_{1}, a_{2}$ be two fresh names of sort $\tau$. We apply Lemma 15 on the equation $T={ }_{E} U$ successively with cut $_{T, a_{1}}$ and cut $_{U^{\prime}, a_{2}}$ where $U^{\prime}=\operatorname{cut}_{T, a_{1}}(U)=f\left(\operatorname{cut}_{T, a_{1}}\left(U_{1}\right), \ldots \operatorname{cut}_{T, a_{1}}\left(U_{n}\right)\right)$. We obtain $a_{1}={ }_{E} a_{2}$, hence $\tau$ is degenerated; contradiction.

2. Assume $f\left(T_{1}, \ldots, T_{n}\right)={ }_{E} U$. Then by Lemma 15 , since $f$ does not occur in $U$, we obtain $a={ }_{E} U$ for some fresh name $a$, hence $\tau$ is degenerated; contradiction.

We are now ready to prove our propositions.

Proposition 17. Let $T_{1}, T_{2}$ be two terms of sort s such that $T_{1} \neq_{E} T_{2}$. Assume a free symbol $\mathrm{h}_{s}: s \times$ Key $\rightarrow$ Hash such that the sort Key is not degenerated. Consider the frame $\varphi_{1}=\left\{x_{1}=\mathrm{h}_{s}\left(T_{1}, k\right), x_{2}=\mathrm{h}_{s}\left(T_{2}, k\right)\right\}$ where $k$ is a fresh name. Let $\varphi_{2}=\left\{x_{1}=n, x_{2}=n^{\prime}\right\}$ where $n, n^{\prime}$ are two distinct fresh names of sort Hash. Then we have $\varphi_{1} \approx_{E} \varphi_{2}$.

Proof. Let $M$ and $N$ be two terms such that $\operatorname{var}(M, N) \subseteq \operatorname{dom}(\varphi)$ and $\operatorname{names}(M, N) \cap \operatorname{names}(\varphi)=\emptyset$.

Assume $M \varphi_{2}={ }_{E} N \varphi_{2}$. Let $\theta$ be the substitution $\left\{n \mapsto \mathrm{h}_{s}\left(T_{1}, k\right), n^{\prime} \mapsto\right.$ $\left.\mathrm{h}_{s}\left(T_{2}, k\right)\right\}$. Since the equational theory $E$ is stable by substitution of names, we have $M \varphi_{2} \theta={ }_{E} N \varphi_{2} \theta$, hence, $M \varphi_{1}={ }_{E} N \varphi_{1}$ as $n, n^{\prime}$ are fresh names.

Conversely, assume $M \varphi_{1}={ }_{E} N \varphi_{1}$. Let $U_{1}=\mathrm{h}_{s}\left(T_{1}, k\right)$. By Lemma 15, we have $\operatorname{cut}_{U_{1}, n}\left(M \varphi_{1}\right)={ }_{E} \operatorname{cut}_{U_{1}, n}\left(N \varphi_{1}\right)$. Since $k$ does not appear in $M$ nor $N$, by Corollary 16, it holds that $\operatorname{cut}_{U_{1}, n}\left(M \varphi_{1}\right)=M \operatorname{cut}_{U_{1}, n}\left(\varphi_{1}\right)$ and $\operatorname{cut}_{U_{1}, n}\left(N \varphi_{1}\right)=$ $N \operatorname{cut}_{U_{1}, n}\left(\varphi_{1}\right)$. Now, using $T_{1} \neq_{E} T_{2}$, we prove $\operatorname{cut}_{U_{1}, n}\left(\varphi_{1}\right)=\left\{x_{1}=n, x_{2}=\right.$ $\left.\mathrm{h}_{s}\left(T_{2}, k\right)\right\}$. Indeed, we have $\operatorname{cut}_{U_{1}, n}\left(\mathrm{~h}_{s}\left(T_{2}, k\right)\right)=\mathrm{h}_{s}\left(\operatorname{cut}_{U_{1}, n}\left(T_{2}\right), k\right)$ since $T_{1} \neq E$ $T_{2}$. Besides, as $k$ does not appear in $T_{2}$, by Corollary 16 , we have cut $_{U_{1}, n}\left(T_{2}\right)=$ $T_{2}$. Similarly, by applying cut $U_{2}, n^{\prime}$ with $U_{2}=\mathrm{h}_{s}\left(T_{2}, k\right)$, we obtain

$$
M \operatorname{cut}_{U_{2}, n^{\prime}}\left(\operatorname{cut}_{U_{1}, n}\left(\varphi_{1}\right)\right)={ }_{E} N \operatorname{cut}_{U_{2}, n^{\prime}}\left(\operatorname{cut}_{U_{1}, n}\left(\varphi_{1}\right)\right),
$$

that is, $M \varphi_{2}={ }_{E} N \varphi_{2}$.

Proposition 18. Let $\varphi$ be a frame and $T$ a term of sort s. Assume a free symbol $\mathrm{h}_{s}: s \times$ Key $\rightarrow$ Hash such that the sort Key is not degenerated. Let $\varphi_{1}=\varphi \cup\left\{x=\mathrm{h}_{s}(T, k), y=k\right\}$ and $\varphi_{2}=\varphi \cup\{x=n, y=k\}$ where $x, y$ are fresh variables, $k$ is a fresh name of sort Key, $n$ is a fresh name of sort Hash. If $\varphi \nvdash_{E} T$, then $\varphi_{1} \approx_{E} \varphi_{2}$.

Proof. Let $M$ and $N$ be two terms such that $\operatorname{var}(M, N) \subseteq \operatorname{dom}(\varphi)$ and names $(M, N) \cap \operatorname{names}(\varphi)=\emptyset$. We prove that $M \varphi_{2}={ }_{E} N \varphi_{2}$ implies $M \varphi_{1}={ }_{E}$ $N \varphi_{1}$ similarly as for Proposition 17 .

Conversely, assume $M \varphi_{1}={ }_{E} N \varphi_{1}$. Let $U=\mathrm{h}_{s}(T, k)$. By Lemma 15, we have $\operatorname{cut}_{U, n}\left(M \varphi_{1}\right)={ }_{E} \operatorname{cut}_{U, n}\left(N \varphi_{1}\right)$. Let us prove that $\operatorname{cut}_{U, n}\left(M \varphi_{1}\right)=$ $M \operatorname{cut}_{U, n}\left(\varphi_{1}\right)$. 
Indeed, otherwise, there exists a subterm $M_{1}$ of $M$ such that $M_{1}$ is not a variable and $M_{1} \varphi_{1}=\mathrm{h}_{s}\left(T^{\prime}, T^{\prime \prime}\right)$ with $T^{\prime}={ }_{E} T$ and $T^{\prime \prime}=_{E} k$. Since $M_{1}$ is not a variable, $M_{1}$ is of the form $M_{1}=\mathrm{h}_{s}\left(M_{1}^{\prime}, M_{1}^{\prime \prime}\right)$ with $M_{1}^{\prime} \varphi_{1}=T^{\prime}={ }_{E} T$, which implies that $T$ is deducible; contradiction.

We deduce that $\operatorname{cut}_{U, n}\left(M \varphi_{1}\right)=M \operatorname{cut}_{U, n}\left(\varphi_{1}\right)$, and similarly $\operatorname{cut}_{U, n}\left(N \varphi_{1}\right)=$ $N \operatorname{cut}_{U, n}\left(\varphi_{1}\right)$. Thus $M \operatorname{cut}_{U, n}\left(\varphi_{1}\right)={ }_{E} N \operatorname{cut}_{U, n}\left(\varphi_{1}\right)$. By Corollary 16, as $k$ does not appear in $\varphi$, we have that $\operatorname{cut}_{U, n}(\varphi)=\varphi$, hence $\operatorname{cut}_{U, n}\left(\varphi_{1}\right)=\varphi_{2}$ and $M \varphi_{2}={ }_{E} N \varphi_{2}$.

Proposition 19. Let $T_{1}, T_{2}$ be two terms of sort s such that $T_{1}=_{E} T_{2}$. Assume a free symbol $\mathrm{h}_{s}: s \times$ Key $\rightarrow$ Hash such that Key is not degenerated. Let $\varphi=\left\{x_{1}=\mathrm{h}_{s}\left(T_{1}, k\right), x_{2}=\mathrm{h}_{s}\left(T_{2}, k\right)\right\}$. Then, $\varphi \approx_{E}\left\{x_{1}=n, x_{2}=n\right\}$ where $n$ is a fresh name of sort Hash.

Proof. Let $M$ and $N$ be two terms such that $\operatorname{var}(M, N) \subseteq \operatorname{dom}(\varphi)$ and $\operatorname{names}(M, N) \cap \operatorname{names}(\varphi)=\emptyset$. We prove that $M \varphi_{2}={ }_{E} N \varphi_{2}$ implies $M \varphi_{1}={ }_{E}$ $N \varphi_{1}$ similarly as for Proposition 17.

Conversely, assume $M \varphi_{1}={ }_{E} N \varphi_{1}$. Let $U=\mathrm{h}_{s}\left(T_{1}, k\right)$. By Lemma 15, we have $\operatorname{cut}_{U, n}\left(M \varphi_{1}\right)={ }_{E} \operatorname{cut}_{U, n}\left(N \varphi_{1}\right)$. Since $k$ does not appear in $M$ nor $N$, by Corollary 16, we have $\operatorname{cut}_{U, n}\left(M \varphi_{1}\right)=\operatorname{Mcut}_{U, n}\left(\varphi_{1}\right)$ and $\operatorname{cut}_{U, n}\left(N \varphi_{1}\right)=$ $\operatorname{Ncut}_{U, n}\left(\varphi_{1}\right)$. Now, since $T_{1}={ }_{E} T_{2}$, we obtain $\operatorname{cut}_{U, n}\left(\varphi_{1}\right)=\left\{x_{1}=n, x_{2}=\right.$ $n\}=\varphi_{2}$. Thus we have $M \varphi_{2}={ }_{E} N \varphi_{2}$.

\section{B. Static equivalence in groups}

We establish some properties of static equivalence in the equational theory of Abelian groups $E_{\mathrm{G}}$ defined in Section 3.2. For this purpose we characterize equivalence classes in $E_{\mathrm{G}}$ by a representation lemma.

Let $\mathcal{X}_{A}\left(\mathcal{X}_{G}\right.$ and $\mathcal{X}_{\text {Hash }}$ respectively) be the set of variables of sort $A$ ( $G$ and Hash respectively). Let $\mathcal{N}_{A}\left(\mathcal{N}_{G}\right.$ and $\mathcal{N}_{\text {Hash }}$ respectively) be the set of names of sort $A$ ( $G$ and Hash respectively). Let $A C$ be the equational theory corresponding to the subset of equations from $E_{\mathrm{G}}$, modeling the associativity and commutativity of the three operators $\cdot,+$ and $*$.

We call unitary monomial of sort $A$ a function $\beta: \mathcal{X}_{A} \cup \mathcal{N}_{A} \rightarrow \mathbb{N}$ almost everywhere zero, i.e., except for a finite number of entries. Such a function $\beta$ can be considered as a term of sort $A$ (modulo $A C)$ :

$$
\beta={ }_{A C} \prod_{a \in \mathcal{N}_{A}, \beta(a) \neq 0} a^{\beta(a)} \cdot \prod_{u \in \mathcal{X}_{A}, \beta(u) \neq 0} u^{\beta(u)}
$$

where empty products are considered to be the term $1_{A}$, and $a^{\beta(a)}(\beta(a) \neq 0)$ denotes the term $\underbrace{a \cdot \ldots \cdot a}_{\beta(a) \text { times }}$. We denote $\mathcal{M}_{A}$ the set of all unitary monomials of sort $A$. 
A canonical form of sort $A$ is a function $\alpha: \mathcal{M}_{A} \rightarrow \mathbb{Z}$ almost everywhere zero. We consider such a function $\alpha$ as a term of sort $A$ (modulo $A C$ ):

$$
\alpha={ }_{A C} \sum_{\beta \in \mathcal{M}_{A}, \alpha(\beta) \neq 0} \alpha(\beta) \cdot \beta
$$

where empty sums are considered to be the term $0_{A}$, and integers are naturally represented as $0_{A}, 1_{A}+\ldots+1_{A}$ or $-\left(1_{A}+\ldots+1_{A}\right)$ of sort $A$.

A canonical form of sort $G$ is a function $\gamma$, mapping terms in $\mathcal{X}_{N} \cup \mathcal{N}_{N}$ to canonical forms of sort $A$, almost everywhere zero, i.e., the function evaluates to the constant 0 except for a finite number of entries. We consider a canonical form $\gamma$ to be a term of sort $G$ (modulo $A C)$ :

$$
\gamma={ }_{A C} \prod_{g \in \mathcal{N}_{G}, \gamma(g) \neq 0} g^{\gamma(g)} * \prod_{x \in \mathcal{X}_{G}, \gamma(x) \neq 0} x^{\gamma(x)}
$$

where empty products are considered to be equal to $1_{G}$.

A canonical form of sort Hash, denoted $\iota$, is either a variable of sort Hash : $\iota=z \in \mathcal{X}_{\text {Hash }}$, a name of sort Hash $: \iota=h \in \mathcal{N}_{\text {Hash }}$ or a canonical form $\gamma$ of sort $G$ considered to be a term $\iota=\mathrm{h}(\gamma)$.

Lemma 20. For any term $T$ of sort $A$ ( $G$, Hash, respectively), there exists a unique canonical form $\alpha_{T}\left(\gamma_{T}, \iota_{T}\right.$, respectively) such that

$$
T={ }_{E_{\mathrm{G}}} \alpha_{T}
$$

$\left(T={ }_{E_{\mathrm{G}}} \gamma_{T}, T={ }_{E_{\mathrm{G}}} \iota_{T}\right.$, respectively $)$.

Proof (Sketch). We show the existence of a canonical form of a term $T$ by induction on the structure of $T$. For instance, given $T=T_{1} * T_{2}$, and two canonical forms $\alpha_{T_{1}}$ and $\alpha_{T_{2}}$, we obtain the canonical form of $T$ by rearranging the product $\alpha_{T_{1}} * \alpha_{T_{2}}$ modulo $E_{\mathrm{G}}$ (and if necessary the induction hypthesis is also used on the exponents). To show the uniqueness of the normal form, it is sufficient to show that whenever two canonical terms are equal as terms modulo $E_{\mathrm{G}}$, they are also equal "mathematically". Formally this is established by studying the $A C$ normal form of each canonical form with respect to the following $A C$-convergent rewriting system.

$$
\begin{aligned}
u+0_{A} & \rightarrow u \\
u+(-u) & \rightarrow 0_{A} \\
u \cdot 1_{A} & \rightarrow u \\
(u+v) \cdot w & \rightarrow u \cdot w+v \cdot w \\
u \cdot 0_{A} & \rightarrow 0_{A} \\
-(u+v) & \rightarrow(-u)+(-v) \\
(-u) \cdot v & \rightarrow-(u \cdot v) \\
-(-u) & \rightarrow u \\
-0_{A} & \rightarrow 0_{A}
\end{aligned}
$$

$$
\begin{aligned}
x * 1_{G} & \rightarrow x \\
\left(x^{u}\right)^{v} & \rightarrow x^{(u \cdot v)} \\
x^{u} * x^{v} & \rightarrow x^{u+v} \\
x^{1_{A}} & \rightarrow x \\
x^{0_{A}} & \rightarrow 1_{G} \\
(x * y)^{u} & \rightarrow x^{u} * y^{u} \\
x * x & \rightarrow x^{\left(1_{A}+1_{A}\right)} \\
x * x^{u} & \rightarrow x^{u+1_{A}} \\
\left(1_{G}\right)^{u} & \rightarrow 1_{G}
\end{aligned}
$$


This rewriting system has been obtained by orienting and completing the equations generating $E_{\mathrm{G}}$, except $A C$, using the tool Cime [40].

Proposition 21. Let $\varphi_{1}=\nu g, a, b .\left\{x_{1}=g, x_{2}=g^{a}, x_{3}=g^{b}, x_{4}=g^{a \cdot b}\right\}$ and $\varphi_{2}=\nu g, a, b, c .\left\{x_{1}=g, x_{2}=g^{a}, x_{3}=g^{b}, x_{4}=g^{c}\right\}$. We have that $\varphi_{1} \approx_{E_{\mathrm{G}}} \varphi_{2}$.

Proof. Let $M, N$ be two terms of the same sort such that $\operatorname{var}(M, N) \subseteq$ $\operatorname{dom}\left(\varphi_{1}\right)$ and names $(M, N) \cap \operatorname{names}\left(\varphi_{1}, \varphi_{2}\right)=\emptyset$.

Assume $M \varphi_{2}={ }_{E_{\mathrm{G}}} N \varphi_{2}$. Let $\theta$ be the substitution $\{c \mapsto a \cdot b\}$. Since the equational theory $E$ is stable by substitution of names, we have $M \varphi_{2} \theta={ }_{E}$ $N \varphi_{2} \theta$, that is, $M \varphi_{1}={ }_{E_{\mathrm{G}}} N \varphi_{1}$ since $c \notin \operatorname{names}(M, N)$.

Conversely, assume $M \varphi_{1}={ }_{E_{G}} N \varphi_{1}$. If $M$ and $N$ are of sort $A$, then $\operatorname{var}(M, N)=\emptyset$ and hence $M \varphi_{2}=M \varphi_{1}={ }_{E} N \varphi_{1}=N \varphi_{2}$.

Otherwise, $M$ and $N$ are of sort $G$. As $M \varphi_{1}={ }_{E_{\mathrm{G}}} N \varphi_{1}$ is equivalent to $M \varphi_{1} *\left(N \varphi_{1}\right)^{-1_{A}}=1_{G}$, we suppose that $N=1_{G}$.

As $\operatorname{var}(M) \subseteq \operatorname{dom}\left(\varphi_{1}\right)$ and names $(M) \cap \operatorname{names}\left(\varphi_{1}, \varphi_{2}\right)=\emptyset$, the canonical form $\gamma$ of $M$ is of the form

$$
M={ }_{E_{\mathrm{G}}} \prod_{g^{\prime} \neq g} g^{\gamma\left(g^{\prime}\right)} * x_{1}^{\gamma\left(x_{1}\right)} * \ldots * x_{4}^{\gamma\left(x_{4}\right)}
$$

where $\gamma\left(g^{\prime}\right)$ and $\gamma\left(x_{i}\right)$ represent closed terms with disjoint names $\{a, b, c\}$. Hence, we have that

$$
M \varphi_{1}={ }_{E_{\mathrm{G}}} \prod_{g^{\prime} \neq g} g^{\prime \gamma\left(g^{\prime}\right)} * g^{\gamma\left(x_{1}\right)+\gamma\left(x_{2}\right) \cdot a+\gamma\left(x_{3}\right) \cdot b+\gamma\left(x_{4}\right) a \cdot b}={ }_{E_{\mathrm{G}}} 1_{G}
$$

and we conclude that for any $i, \gamma\left(x_{i}\right)=0_{A}$ and for any $g^{\prime}, \gamma\left(g^{\prime}\right)=0_{A}$, i.e., $M=1_{G}$.

Proposition 22. Let the frame $\varphi_{1}=\nu g, a \cdot\left\{x_{1}=g^{a}, x_{2}=a, x_{3}=\mathrm{h}(g)\right\}$ and the frame $\varphi_{2}=\nu g, a, h .\left\{x_{1}=g^{a}, x_{2}=a, x_{3}=h\right\}$. We have that $\varphi_{1} \approx_{E_{\mathrm{G}}} \varphi_{2}$.

Proof. Let $M, N$ be two terms such that $\operatorname{var}(M, N) \subseteq \operatorname{dom}\left(\varphi_{1}\right)$ and $\operatorname{names}(M, N) \cap$ $\operatorname{names}\left(\varphi_{1}, \varphi_{2}\right)=\emptyset$.

Assume $M \varphi_{2}={ }_{E} N \varphi_{2}$. Let $\theta$ be the substitution $\{h \mapsto \mathrm{h}(g)\}$. Since the equational theory $E$ is stable by substitution of names, we have $M \varphi_{2} \theta={ }_{E}$ $N \varphi_{2} \theta$, hence, as $h \notin \operatorname{names}(M, N), M \varphi_{1}={ }_{E} N \varphi_{1}$.

Conversely, assume that $M \varphi_{1}={ }_{E_{G}} N \varphi_{1}$. If $M$ and $N$ are of sort $A$ or $G$, then $\operatorname{var}(M, N) \subseteq\left\{x_{1}, x_{2}\right\}$ and hence $M \varphi_{2}=M \varphi_{1}={ }_{E} N \varphi_{1}=N \varphi_{2}$.

Otherwise, $M$ and $N$ are of sort Hash. We suppose that $M=x_{3}$ and $N=\mathrm{h}\left(N^{\prime}\right)$ where $\operatorname{var}\left(N^{\prime}\right) \subseteq\left\{x_{1}, x_{2}\right\}$ (other cases are trivial). As $\mathrm{h}$ is a free symbol, by Corollary $16, M \varphi_{1}={ }_{E_{\mathrm{G}}} N \varphi_{1}$ is equivalent to $N^{\prime} \varphi_{1}={ }_{E_{\mathrm{G}}} g$.

Given that $\operatorname{var}\left(N^{\prime}\right) \subseteq\left\{x_{1}, x_{2}\right\}$ and names $\left(N^{\prime}\right) \cap \operatorname{names}\left(\varphi_{1}, \varphi_{2}\right)=\emptyset$, the canonical form $\gamma$ of $N^{\prime}$ is of the form

$$
N^{\prime}=E_{\mathrm{G}} \prod_{g^{\prime} \neq g} g^{\prime \gamma\left(g^{\prime}\right)} * x_{1}^{\gamma\left(x_{1}\right)}
$$


where $\gamma\left(g^{\prime}\right)$ and $\gamma\left(x_{1}\right)$ are terms that have no variable other than $x_{2}$ and do not contain $a$. Hence we have

$$
N^{\prime} \varphi_{1}={ }_{E_{\mathrm{G}}} \prod_{g^{\prime} \neq g} g^{\prime \gamma\left(g^{\prime}\right)\left\{x_{2} \mapsto a\right\}} * g^{\gamma\left(x_{1}\right) \cdot a}
$$

which contradicts $N^{\prime} \varphi_{1}={ }_{E_{\mathrm{G}}} g$.

\section{Static equivalence in ciphers and lists}

Before proving Lemma 10, we first introduce a handy lemma to characterize deducible terms.

Lemma 23. Let $\varphi=\nu \tilde{n}$. $\sigma$ be a closed frame in $\mathcal{R}$-normal form and $T$ a term in $\mathcal{R}$-normal form. If $\varphi \vdash_{E_{\text {sym }}} T$ then $T=C\left[T_{1}, \ldots, T_{k}\right]$ where the $T_{i}$ are deducible subterms of $\varphi$ and $C$ is a context that does not contain private names that is $\operatorname{names}(C) \cap \tilde{n}=\emptyset$.

Proof. By definition, $\varphi \vdash_{E_{\text {sym }}} T$ if and only if there exists a term $M$ such that $\operatorname{names}(M) \cap \operatorname{names}(\varphi)=\emptyset$ and $M \varphi={ }_{E_{\text {sym }}} T$, that is, $M \varphi \rightarrow_{\mathcal{R}}^{*} T$. We prove Lemma 23 by induction on the size of $M$. The base case $M=x_{i}$ is trivial.

If $M=f\left(M_{1}, \ldots, M_{k}\right)$. We only consider the case where $M=\operatorname{dec}\left(M_{1}, M_{2}\right)$ since the other cases are similar. We have $M_{1} \rightarrow_{\mathcal{R}}^{*} T_{1}$ and $M_{2} \rightarrow_{\mathcal{R}}^{*} T_{2}$. By applying the induction hypothesis to $M_{1}$ and $M_{2}$, we obtain that $T_{1}=C_{1}\left[T_{1}^{\prime}, \ldots, T_{k}^{\prime}\right]$ and $T_{2}=C_{2}\left[T_{1}^{\prime}, \ldots, T_{k}^{\prime}\right]$ where the $T_{i}^{\prime}$ are deducible subterms of $\varphi$ and $C_{1}, C_{2}$ are contexts that do not contain names. We have $M \varphi \rightarrow_{\mathcal{R}}^{*} \operatorname{dec}\left(T_{1}, T_{2}\right)$. Either $\operatorname{dec}\left(T_{1}, T_{2}\right)$ is in $\mathcal{R}$-normal form. In that case and by convergence of $\mathcal{R}$, we have $T=\operatorname{dec}\left(T_{1}, T_{2}\right)$, hence the result. Or $\operatorname{dec}\left(T_{1}, T_{2}\right)$ is not in $\mathcal{R}$-normal form. By convergence, we have $\operatorname{dec}\left(T_{1}, T_{2}\right) \rightarrow_{\mathcal{R}} T$. Since $T_{1}$ and $T_{2}$ are already in normal form, we must have $T_{1}=\operatorname{enc}\left(T_{1}^{\prime}, T_{2}\right)$ and $T=T_{1}^{\prime}$. Either $C_{1}=\operatorname{enc}\left(C_{1}^{\prime}, C_{1}^{\prime \prime}\right)$ and we have $T=C_{1}^{\prime}\left[T_{1}^{\prime}, \ldots, T_{k}^{\prime}\right]$. Or $C_{1}=\ldots$, which means that $T_{1}$ is a deducible subterm of $\varphi$. We deduce that $T$ is a deducible subterm of $\varphi$, hence the result.

We can now start the proof of Lemma 10.

Proof. In what follows, we say that a term or a context is public if it does not contain the names occurring in $\varphi$. Since $\varphi=\varphi^{\prime}\{n \mapsto T\}$ and $E_{\text {sym }}$ is stable by substitutions of names, we have $\operatorname{eq}_{E_{\text {sym }}}\left(\varphi^{\prime}\right) \subseteq \operatorname{eq}_{E_{\text {sym }}}(\varphi)$. To prove $\operatorname{eq}_{E_{\text {sym }}}(\varphi) \subseteq \operatorname{eq}_{E_{\text {sym }}}\left(\varphi^{\prime}\right)$, we introduce the following lemma. We set $\theta$ to be $\{n \mapsto T\}$. Let $n_{1}, \ldots, n_{p}$ be the names occurring in $\varphi^{\prime}$.

Lemma 24. Let $C_{1}$ be a context such that we have $\varphi^{\prime} \vdash_{E_{\text {sym }}} C_{1}\left[n_{1}, \ldots, n_{p}\right]$ and $C_{1}\left[n_{1}, \ldots, n_{p}\right] \theta \rightarrow_{\mathcal{R}} T$. Then there exists a public context $C_{2}$ such that $C_{1} \rightarrow_{\mathcal{R}} C_{2}$ and $T=C_{2}\left[n_{1}, \ldots, n_{p}\right] \theta$. 
The lemma is proved by inspection of the rules of $\mathcal{R}$. The reduction occurs at some position $p$ : the reduction $\left.C_{1}\left[n_{1}, \ldots, n_{p}\right]\right|_{p} \theta \rightarrow_{\mathcal{R}} T$ occurs in head. Let $C_{1}^{\prime}\left[n_{1}, \ldots, n_{p}\right]=\left.C_{1}\left[n_{1}, \ldots, n_{p}\right]\right|_{p}$ If $C_{1}^{\prime}$ is itself an instance of the lefthand-side of a rule of $\mathcal{R}$, than we clearly have that $C_{1}^{\prime} \rightarrow_{\mathcal{R}} C_{2}^{\prime}$ such that $T=C_{2}\left[n_{1}, \ldots, n_{p}\right] \theta$, where $C_{2}$ is obtained from $C_{1}$ by replacing $C_{1}^{\prime}$ with $C_{2}^{\prime}$ at position $p$. If $C_{1}^{\prime}$ is not an instance of the left-hand-side of a rule of $\mathcal{R}$ and since $T$ is already in $\mathcal{R}$-normal form, there are only four possibilities for $C_{1}^{\prime}\left[n_{1}, \ldots, n_{p}\right]$.

- $C_{1}^{\prime}\left[n_{1}, \ldots, n_{p}\right]=\operatorname{enc}\left(n_{i}, C_{1}^{\prime \prime}\left[n_{1}, \ldots, n_{p}\right]\right)$. It must be the case that $n_{i}=n$, $T$ is of the form $\operatorname{dec}(U, V)$ and $V=C_{1}^{\prime \prime}\left[n_{1}, \ldots, n_{p}\right]$. From Lemma 23 and since $\varphi^{\prime} \vdash_{E_{\text {sym }}} C_{1}\left[n_{1}, \ldots, n_{p}\right]$, either $C_{1}^{\prime}\left[n_{1}, \ldots, n_{p}\right]$ is subterm of $\varphi^{\prime}$ or $n_{i}$ and $C_{1}^{\prime \prime}\left[n_{1}, \ldots, n_{p}\right]$ are deducible. In both cases, we obtain a contradiction. Indeed, if $C_{1}^{\prime}\left[n_{1}, \ldots, n_{p}\right]$ is subterm of $\varphi^{\prime}$ then $C_{1}^{\prime}\left[n_{1}, \ldots, n_{p}\right] \theta=$ enc $\left(\operatorname{dec}(U, V), n_{j}\right)$ is a subterm of $\varphi$, which contradicts that $\varphi$ is in normal form. If $n_{i}$ and $C_{1}^{\prime \prime}\left[n_{1}, \ldots, n_{p}\right]$ are deducible then this contradicts $\varphi \nvdash_{E_{\text {sym }}} V$.

- $C_{1}^{\prime}\left[n_{1}, \ldots, n_{p}\right]=\operatorname{dec}\left(n_{i}, n_{j}\right)$. This case is very similar to the previous one.

- $C_{1}^{\prime}\left[n_{1}, \ldots, n_{p}\right]=\operatorname{cons}\left(n_{i}, C_{1}^{\prime \prime}\left[n_{1}, \ldots, n_{p}\right]\right)$. It must be the case that $n_{i}=n$, $T$ is of the form head $(V)$ and $C_{1}^{\prime \prime}\left[n_{1}, \ldots, n_{p}\right]=\operatorname{tail}(V)$. From Lemma 23 and since $\varphi^{\prime} \vdash_{E_{\text {sym }}} C_{1}\left[n_{1}, \ldots, n_{p}\right]$, either $C_{1}^{\prime}\left[n_{1}, \ldots, n_{p}\right]$ is subterm of $\varphi^{\prime}$ or $n_{i}$ and $C_{1}^{\prime \prime}\left[n_{1}, \ldots, n_{p}\right]$ are deducible. As previously, in both cases, we obtain a contradiction. if $C_{1}^{\prime}\left[n_{1}, \ldots, n_{p}\right]$ is subterm of $\varphi^{\prime}$ then $C_{1}^{\prime}\left[n_{1}, \ldots, n_{p}\right] \theta=$ $\operatorname{cons}(\operatorname{head}(V)$, tail $(V))$ is a subterm of $\varphi$, which contradicts that $\varphi$ is in normal form. If $n_{i}$ and $C_{1}^{\prime \prime}\left[n_{1}, \ldots, n_{p}\right]$ are deducible then both $n$ and tail $(V)$ are deducible in $\varphi^{\prime}$, which means that both head $(V)$ and tail $(v)$ are deducible in $\varphi$, thus $V$ is deducible in $\varphi$, contradiction.

- $C_{1}^{\prime}\left[n_{1}, \ldots, n_{p}\right]=\operatorname{cons}\left(C_{1}^{\prime \prime}\left[n_{1}, \ldots, n_{p}\right], n_{i}\right)$. This case is very similar to the previous one.

Now, let $(M=N) \in \mathrm{eq}_{E_{\text {sym }}}(\varphi)$ and let us show that $(M=N) \in \operatorname{eq}_{E_{\text {sym }}}\left(\varphi^{\prime}\right)$. We have $M \varphi=E_{E_{\text {sym }}} N \varphi$, that is, $M \varphi^{\prime} \theta=E_{E_{\text {sym }}} N \varphi^{\prime} \theta$. By convergence of $\mathcal{R}$, there exists a term $T$ such that $M \varphi^{\prime} \theta \rightarrow_{\mathcal{R}}^{*} T$ and $N \varphi^{\prime} \theta \rightarrow_{\mathcal{R}}^{*} T$. By applying repeatedly Lemma 24 , we obtain that $M \varphi^{\prime} \rightarrow_{\mathcal{R}}^{*} T_{1}$ such that $T=T_{1} \theta$ and $N \varphi^{\prime} \rightarrow_{\mathcal{R}}^{*} T_{2}$ such that $T=T_{2} \theta$. Assume that we have proved that $T_{1}=T_{2}$. Then we have $M \varphi^{\prime}={ }_{E_{\text {sym }}} N \varphi^{\prime}$, that is, $(M=N) \in \mathrm{eq}_{E_{\text {svm }}}\left(\varphi^{\prime}\right)$, which concludes the proof. It remains for us to prove the following lemma.

Lemma 25. Let $T_{1}$ and $T_{2}$ be two terms such that each $T_{i}$ is either deducible from $\varphi^{\prime}$, that is, $\varphi^{\prime} \vdash_{E_{\text {sym }}} T_{i}$, or $T_{i}$ is a subterm of $\varphi^{\prime}$. Then $T_{1} \theta=T_{2} \theta$ implies $T_{1}=T_{2}$.

The lemma is proved by induction on the sum of the size of $T_{1}$ and $T_{2}$. First notice that, by Lemma 23, any subterm $T^{\prime}$ of one of the $T_{i}$ verifies that $T^{\prime}$ is deducible from $\varphi^{\prime}$ or $T^{\prime}$ is a subterm of $\varphi^{\prime}$. 
- The base case is trivial.

- If none of $T_{1}$ or $T_{2}$ is $n: T_{1}=f\left(T_{1}^{\prime}, \ldots, T_{k}^{\prime}\right)$ and $T_{2}=f\left(T_{1}^{\prime \prime}, \ldots, T_{k}^{\prime \prime}\right)$. We must have $T_{i}^{\prime} \theta=T_{i}^{\prime \prime} \theta$ for every $1 \leq i \leq k$. By applying the induction hypothesis, we obtain $T_{i}^{\prime}=T_{i}^{\prime \prime}$ thus $T_{1}=T_{2}$.

- The most difficult case is when $T_{1}=n$ and $T_{2}=f\left(T_{1}^{\prime}, \ldots, T_{k}^{\prime}\right)$. We first notice that since $n \theta=f\left(T_{1}^{\prime}, \ldots, T_{k}^{\prime}\right) \theta$, n cannot occur in $T_{2}$, thus $T_{2}=T_{2} \theta$. Either $T_{2}$ is a subterm of $\varphi^{\prime}$, which is impossible by construction of $\varphi^{\prime}$ or $T_{2}$ deducible. Since $T_{2}$ is not a subterm of $\varphi^{\prime}$ and applying again Lemma 23, we get that the immediate subterms of $T_{2}$ are deducible in $\varphi^{\prime}$ (thus in $\varphi$ ), which contradicts the choice of $T$. 